\title{
Flame Double Input Describing Function analysis
}

\author{
Alessandro Orchini*, Matthew P. Juniper \\ Department of Engineering, University of Cambridge, Trumpington Street, Cambridge, CB2 1PZ, UK
}

\begin{abstract}
The Flame Describing Function (FDF) is a useful and relatively cheap approximation of a flame's nonlinearity with respect to harmonic velocity fluctuations. When embedded into a linear acoustic network, it is able to predict the amplitude and stability of harmonic thermoacoustic oscillations through the harmonic balance procedure. However, situations exist in which these oscillations are not periodic, but their spectrum contains peaks at several incommensurate frequencies. If one assumes that two frequencies dominate the spectrum, these oscillations are quasiperiodic, and the FDF concept can be extended by forcing the flame with two amplitudes and two frequencies. The nonlinearity is then approximated by a Flame Double Input Describing Function (FDIDF), which is a more expensive object to calculate than the FDF, but contains more information about the nonlinear response.

In this study, we present the calculation of a non-static flame's FDIDF. We use a $G$-equation-based laminar conical flame. We embed the FDIDF into a thermoacoustic network and we predict the nature and amplitude of thermoacoustic oscillations through the harmonic balance method. A criterion for the stability of these oscillations is outlined. We compare our results with a classical FDF analysis and self-excited time domain simulations of the same system. We show how the FDIDF improves the stability prediction provided by the FDF. At a numerical cost roughly equivalent to that of two FDFs, the FDIDF is capable to predict the onset of Neimark-Sacker bifurcations and to identify the frequency of oscillations around unstable limit cycles. At a higher cost, it can also saturate in amplitude these oscillations and predict the amplitude and stability of quasiperiodic oscillations.
\end{abstract}

Keywords: Flame Describing Function, Premixed flame response, Thermoacoustic oscillations

\section{1. Introduction}

Thermoacoustic oscillations are a persistent problem in rocket and gas turbine engines. While their onset can be modelled with linear methods, prediction of their finite amplitude behaviour requires the use of nonlinear techniques. In the last decade these

* Corresponding author

Email address: ao352@cam.ac.uk (Alessandro Orchini) 


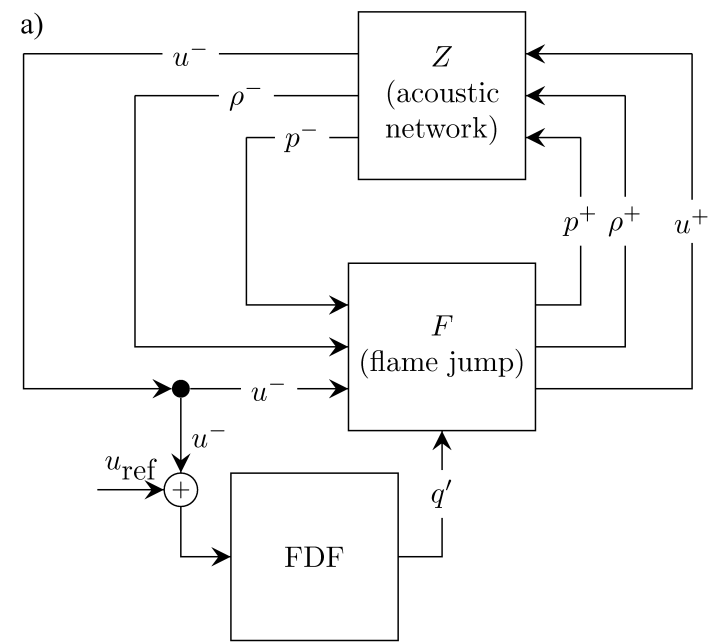

b)

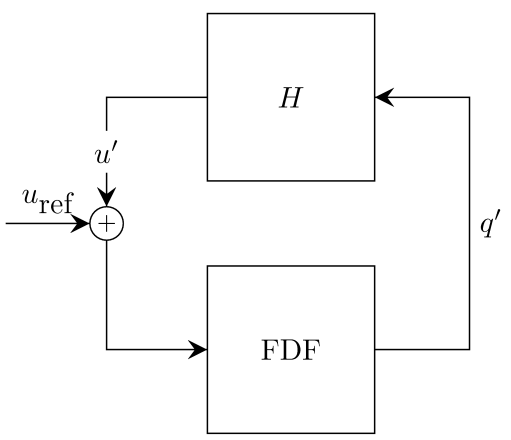

Figure 1: Overview of a closed-loop thermoacoustic network. $\rho, p$ and $u$ denote the flow density and acoustic pressure and velocity variables. (a) The jump across the flame element is highlighted, using superscripts - and + for the acoustic properties upstream and downstream the flame, respectively. All the remaining acoustic information is embedded into the acoustic block $Z$. (b) The same closed-loop thermoacoustic network simplified across the FDF element; $H$ contains all the linear acoustic response with respect to heat release perturbations. nonlinear methods have involved both frequency domain $[1,2,3,4,5,6]$ and time domain methods $[7,8,9,10,11,12]$.

Time domain methods tend to be computationally expensive. One usually converts the (linear) frequency response of a given acoustic system into the time domain by using Green's functions [7, 9, 10], Fourier modes [5, 11] or a state space approach [8, 13, 14]. 10 One then couples this with a nonlinear flame model and performs a simulation forward ${ }_{11}$ in time, exploiting the full nonlinear characteristics of the flame model. However, this is 12 usually expensive, even for low-order models, because many oscillation cycles have to be 13 simulated before the final attractor of the system is reached [11]. Numerical continuation 14 algorithms [12] are cheaper, but require smooth numerical integration techniques. They 15 can predict limit cycle oscillations and their stability, but not the amplitude of nonperiodic oscillations.

On the other hand, frequency domain methods tend to be cheap. Rather than simulate the entire system's nonlinear behaviour, one encapsulates the flame's nonlinear response with a Flame Describing Function (FDF), which is the frequency response of the flame with respect to harmonic forcing at variable amplitude. FDF methods were introduced in thermoacoustics by [15], and first fully exploited by [6]. The calculation of an FDF can be expensive, but if the flame model is not changed, the same FDF can be used to test many acoustic configurations with a low-cost procedure, known as harmonic balance.

For the harmonic balance analysis, the nonlinear flame dynamics is decoupled from the acoustics. This can be done if the flame is acoustically compact, meaning that the characteristic flame length $L_{f}$ is much shorter than the smallest acoustic wavelength of 
interest $\lambda_{\min }=c / f_{\max }$. Under this assumption, a generic thermoacoustic configuration can be drawn as a block diagram as in Fig. 1a. The acoustic jump conditions across the flame have been highlighted. Their inputs are the acoustic variables upstream of the flame and the instantaneous heat release fluctuations, $q^{\prime}$. The acoustics is expressed in terms of downstream $(f)$ and upstream $(g)$ travelling waves. The remaining acoustic response is contained in the upstream and downstream acoustic blocks. For the simple configuration composed of two straight ducts interconnected by a flame, they contain information about the mean flow, end reflection coefficients, and wave time delays [3]. Finally, the FDF converts velocity disturbances upstream of the flame into heat release fluctuations.

The feedback loop in Fig. 1a can be simplified by choosing a reference input signal

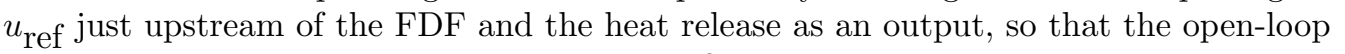
heat release response with respect to velocity fluctuations is given by:

$$
q^{\prime}=\mathrm{FDF}\left(u^{\prime}+u_{\mathrm{ref}}\right)
$$

Furthermore, the entire open-loop acoustic response with respect to heat release fluctuations can be embedded into a unique transfer function $H$ so that, for velocity fluctuations upstream of the flame, we can write (see Fig. 1b):

$$
u^{\prime}=H q^{\prime}
$$

For simple acoustic networks, the expression for the transfer function $H$ can be found analytically $[3,14]$. It becomes rather complicated for complex networks, and numerical methods are used in these cases to evaluate $H$ over a certain range of frequencies.

Closing the feedback loop between the velocity at the reference point and the heat release fluctuations yields:

$$
q^{\prime}=\frac{\operatorname{FDF}(A, s)}{1-\operatorname{FDF}(A, s) H(s)} u_{\mathrm{ref}}
$$

Equation (3) represents a Single Input Single Output system: if no input velocity is prescribed, the system will be linearly unstable if and only if it has poles in the r.h.s. of the complex plane in the zero amplitude limit. Looking for these poles is equivalent to finding solutions to the harmonic balance dispersion relation

$$
\operatorname{FDF}(A, s) H(s)=1
$$

where $A$ is the upstream velocity amplitude and $s=\sigma+i \omega$ is the Laplace variable. The dispersion relation (4) is also able to identify poles which have a negative growth rate at small amplitudes, but become unstable at finite amplitudes. This is a characteristic of subcritical Hopf bifurcations, and phenomena such as bistability and triggering may be observed.

Solving the dispersion relation (4) at various amplitudes leads to harmonic limit cycle solutions of the closed-loop system, for which the growth rate $\sigma$ is equal to zero. Their stability may be analysed by investigating the change in growth rate across the saturated amplitude [6, 14, 16]. These solutions are, however, only harmonic approximations of the actual response of the system. Furthermore, if the growth rate of more than one thermoacoustic mode is positive, then the oscillations are non-periodic. Because the 
FDF is calculated by forcing the flame harmonically, it cannot be used to predict the amplitude of non-periodic oscillations. In particular, one cannot linearly superpose two periodic solutions that are found from the harmonic balance at a given operating point. This is simply because the flame's behaviour when forced by two finite amplitude signals is not a linear superposition of its behaviour when forced by each finite amplitude signal independently. When using the FDF, therefore, one cannot rule out the possibility that the long time behaviour is non-periodic.

A detailed investigation of the interplay between two oscillating modes has been performed experimentally only for simple configurations [17], due to its high cost. Nonetheless, the presence of multiple, incommensurate frequencies in the spectrum of thermoacoustic oscillations has been reported in several experimental studies [18, 19, 20]. The study of the nonlinear interaction between the modes may be relevant for the analysis of these systems. It has also been observed in experiments that, although a single eigenmode is found to be linearly unstable, nonlinear effects may actually stabilise the oscillations at this frequency and trigger oscillations at a different frequency [6]. The FDF can predict the existence of oscillations of the two modes independently, but will fail in predicting their stability, as the latter is connected to the nonlinear coupling between the two modes. This phenomenon is usually called mode-switching, and was observed also by [21] and in gas turbines experiments by $[22,23]$. In [21] it was shown that mode-switching can be attributed to the existence of an unstable quasiperiodic attractor in the phase-space of thermoacoustic trajectories, which the FDF framework cannot calculate.

In order to predict the amplitude of at least some classes of non-periodic oscillations, a different approximation of the nonlinear flame model has to be calculated. This is known as the Double Input Describing Function (DIDF), and is created by forcing the flame with a signal composed of two harmonic components with independent amplitudes and incommensurate frequencies [24]. The calculated Flame DIDF (FDIDF) can then be fed into an acoustic network in a similar manner to that in Fig. 1. The harmonic balance procedure yields two coupled dispersion relations which have to be solved simultaneously, as was first shown by [21] for a thermoacoustic system.

The aim of this study is to present a numerical analysis that exploits frequency domain calculations of a non-static (or dynamic ${ }^{1}$ ) nonlinearity based on a low-order model for the flame dynamics. This is the major difference between our analysis and that of [21], where a static model for the flame was considered. For static nonlinearities, a Wiener-Hammerstein model can be adopted, which decouples the nonlinear amplitude saturation process from the linear dynamic response. This is not possible for dynamic nonlinearities, and the FDIDF we calculate is a nonlinear object that couples the input amplitudes and frequencies. We also obtain an analytical criterion for the stability of quasiperiodic oscillations, which is different from the one discussed in [21]. A different attempt to extend the concept of the FDF was proposed by [25, 26], where higher order transfer functions that account for modal coupling were derived using Volterra series expansions. However, the dependence of the higher order transfer functions upon the relative amplitude of the input modes was not considered in these studies. The non-static model we adopt for the flame is the kinematic nonlinear $G$-equation, which is known to lead to quasiperiodic oscillations when coupled with an acoustic network $[11,12,14]$.

\footnotetext{
1 A nonlinearity is non-static if it depends on time derivatives of the input state.
} 


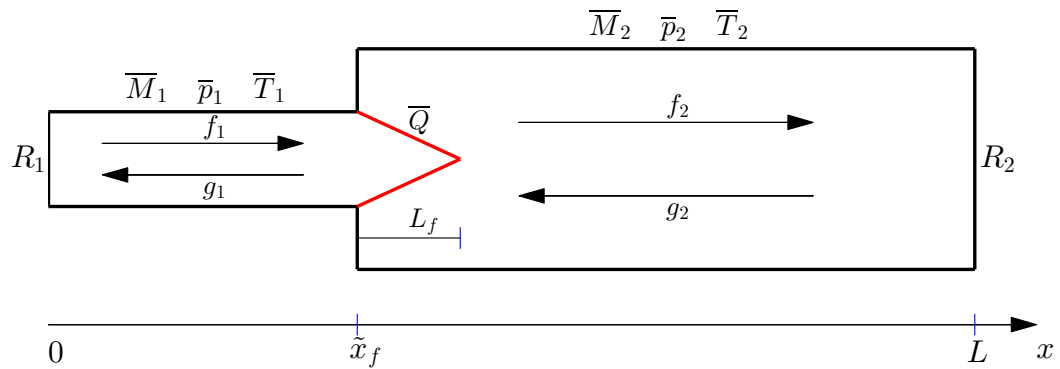

Figure 2: Sketch of the thermoacoustic network. A compact flame connects two ducts with different diameters and mean temperatures. The linearised Euler equations are solved on top of a uniform mean flow solution. Jump conditions at the flame and prescribed reflection coefficients at the inlet/outlet provide closure for the model.

The FDIDF method is able to predict the location of Neimark-Sacker bifurcations, the frequency of unstable oscillations around limit cycles, and also the saturation amplitude and the stability of quasiperiodic oscillations. The study is structured as follows: in $\S 2$ we describe the acoustic and flame configurations we investigate; in $\S 3$ we present FDF results and calculate harmonic limit cycles amplitudes and frequencies, together with their stability, highlighting strong points and weaknesses of the method; in $\S 4$ and $\S 5$ the FDIDF is presented and tested against the FDF in the limit of a small forcing amplitude; the dispersion relations which couple it with the acoustic response are derived and solved; the frequencies and amplitudes of periodic and non-periodic solutions are calculated with the harmonic balance method based on the FDIDF; a criterion for the stability of these solutions is outlined; results are compared with the FDF method analysis and with time domain simulations of the same nonlinear system. Finally in $\S 6$ the study is summarised and the benefits and problems of the methods are discussed.

\section{Thermoacoustic model}

The thermoacoustic model we will consider throughout this study consists of a laminar, conical flame confined in a simple acoustic network. The same model has been presented and extensively discussed in $[14,27]$ but is summarized here for completeness.

\subsection{Acoustic network}

The acoustic network we consider is shown in Fig. 2. It consists of two interconnected ducts with different cross sectional areas. A flame, assumed to be acoustically compact, is located just after the area change. Rankine-Hugoniot jump conditions for mass, momentum and energy are solved across the area change and the flame to guarantee the conservation of these fluxes [3]. Reflection coefficients, which may be frequency dependent, are specified at the inlet and outlet. This is a simple but generic model for a combustion driven Rijke tube, such as the one analysed experimentally by [28].

The geometry and mean flow parameters are: total length of the combustor $L=$ $860 \mathrm{~mm}$; upstream and downstream duct diameters $D_{1}=23 \mathrm{~mm}$ and $D_{2}=25.6 \mathrm{~mm}$; inlet temperature $\bar{T}_{1}=300 \mathrm{~K}$; Mach number upstream of the flame $\bar{M}_{1}=0.0057$; outlet 


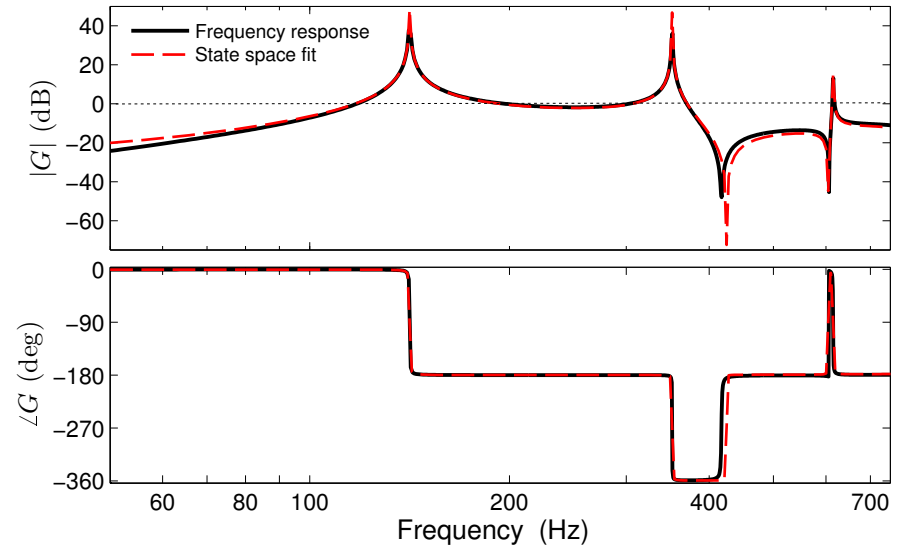

Figure 3: Comparison between the frequency response calculated with LOTAN (solid black) and the state space approximation (dashed red) at $x_{f}=0.34$. The approximation works well over a wide range of frequencies.

pressure equals to atmospheric pressure; temperature ratio across the flame $\bar{T}_{2} / \bar{T}_{1}=$ 2. Note that the average temperature in the downstream duct, $\bar{T}_{2}$, is lower than the adiabatic flame temperature in order to account for heat losses through the walls. We will use the non-dimensional position of the flame in the duct, defined as $x_{f} \equiv \tilde{x}_{f} / L$, where $\tilde{x}_{f}$ is the dimensional flame position value, as a bifurcation parameter. Finally, we use a frequency dependent reflection coefficients at the inlet and outlet when solving the acoustic equations. We choose the low-Mach number limit of the reflection coefficient derived analytically by $[29,30]$ using the Wiener-Hopf technique. It has been validated against experiments in [31]

Using the Low-Order ThermoAcoustic Network (LOTAN) framework [4, 32], the acoustic network is solved in the frequency domain by decomposing the acoustic variables into upstream and downstream travelling waves (see Fig. 2). We calculate the acoustic eigenfrequencies and, by imposing harmonic fluctuations in the heat release at the combustion zone, we evaluate the open-loop acoustic transfer function $H_{x_{f}}$ as in eq. (2). Using both the frequency response and the eigenfrequencies, the acoustic response to heat release fluctuations can be fitted onto a state space, as described in [14]. This is necessary to extend the frequency response - calculated at $s=i \omega-$ in the full Laplace space, in which the growth rate $\sigma$ can be non-zero. Fig. 3 shows that the state space approximation fits well the frequency response evaluated with LOTAN over a wide range of frequencies.

Note that, by moving the flame position, the acoustic response of the system will change, but the flame response will not. Thus, a different acoustic transfer function (and its state space approximation) has to be evaluated each time the bifurcation parameter $x_{f}$ is changed. This, however, is a cheap calculation. On the other hand, only one (expensive) Flame Describing Function calculation has to be performed on the flame. The same FDF can be used to study the stability of the system for any value of the bifurcation parameter. 


\subsection{Flame model}

As a model for the flame, we use the nonlinear kinematic $G$-equation to track the flame front, which is located at the $G=0$ level set [33, 34, 35]. We consider a laminar, conical, axisymmetric flame. The flow field is assumed to be incompressible and, to simplify the calculations, we neglect the density jump across the flame. The local flame speed depends on the local flame curvature $\kappa$ through the relation $s_{L}=s_{L}^{0}(1-\mathcal{L} \kappa)$, where $\mathcal{L}$ is the Markstein length and $s_{L}^{0}$ the speed of a flat, laminar flame. The flow field is composed of a uniform mean axial velocity $\bar{U}$, on top of which forced perturbations are imposed, denoted with primes and described below. The perturbations are specified at the burner inlet $x_{b}$ and then travel at a characteristic velocity $K$ in the flame domain [36]. We fix the value of the convective speed to $K=1.2 \bar{U}$, which is within the range obtained numerically by [37] for a laminar conical flame. Under these assumptions, the $G$-equation model is:

$$
\frac{\partial G}{\partial t}+u_{r}^{\prime} \frac{\partial G}{\partial r}+\left(\bar{U}+u^{\prime}\right) \frac{\partial G}{\partial x}=s_{L}^{0}(1-\kappa \mathcal{L}) \sqrt{\left(\frac{\partial G}{\partial r}\right)^{2}+\left(\frac{\partial G}{\partial x}\right)^{2}}
$$

We solve this equation with the efficient Narrow Band Level Set method technique [38, 39]. We choose an incompressible travelling wave as a model for the perturbation flow [36, 37, 40], which reads:

$$
\begin{array}{cc}
\frac{\partial u^{\prime}}{\partial t}+K \frac{\partial u^{\prime}}{\partial x}=0 & u^{\prime}\left(x=x_{b}\right)=u_{a c}^{\prime}(t) \\
\frac{1}{r} \frac{\partial\left(r u_{r}^{\prime}\right)}{\partial r}+\frac{\partial u^{\prime}}{\partial x}=0 & u_{r}^{\prime}(r=0)=0
\end{array}
$$

Here $u_{a c}^{\prime}$ denotes the acoustic perturbation imposed at the inlet. The total heat release is then given by

$$
Q=\int_{G=0} \rho s_{L}^{0} h_{r}(1-\mathcal{L} \kappa)|\nabla G| r \mathrm{~d} r \mathrm{~d} x
$$

where $\rho$ is the flow density and $h_{r}$ the heat released per unit mass. This $G$-equation based model has been extensively studied both in the linear [27, 41, 42, 43, 44, 45] and nonlinear $[12,14,35,37,46]$ regimes when the imposed perturbations are harmonic, i.e. $u_{a c}^{\prime}=A \sin (\omega t)$. The main goal of this paper is to extend the nonlinear analysis in the frequency domain to the case in which the inlet perturbation is given by the sum of two incommensurate harmonic fluctuations, resulting in quasiperiodic oscillations. Nonetheless, the harmonic case is instructive and its discussion is needed to present some of the assumptions we will use in the quasiperiodic analysis and to benchmark the FDIDF calculations.

\section{FDF analysis}

By FDF, we refer to the frequency domain approximation of the nonlinear flame response to harmonic velocity perturbations (see Figure 4). We therefore set $u_{a c}^{\prime}=A \sin (\omega t)$, time march eq.(5)-(6), and calculate the heat release according to eq. (7). Given that the 

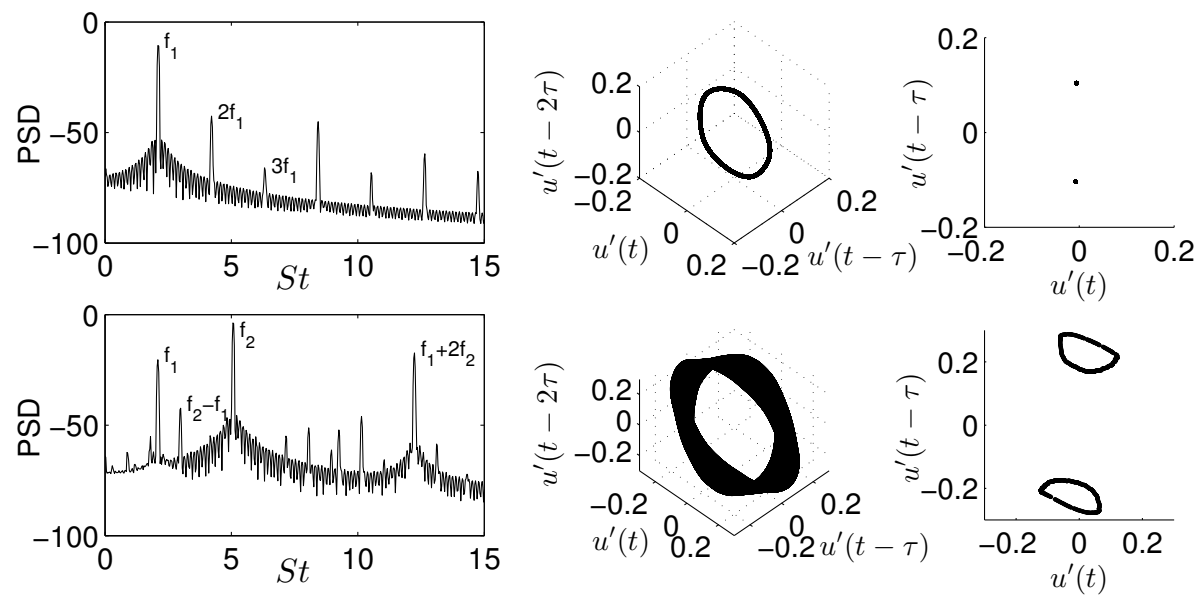

Figure 4: Velocity Power Spectral Density (PSD), phase plane and Poincaré sections of self-excited thermoacoustic oscillations. Top row: periodic oscillations. The system responds also at the harmonics, which are neglected in the FDF framework. Bottom row: quasiperiodic oscillations. The system responds also at the harmonics and linear combination of the fundamental frequencies, which are neglected by the FDIDF. Image reproduced from [14] with permission from Cambridge University Press.

velocity perturbation is harmonic with angular frequency $\omega$, it is reasonable to assume that the heat release response can be expanded in a Fourier series as:

$$
Q=\sum_{k=1}^{\infty} \hat{q}_{k} \sin \left(k \omega t+\phi_{k}\right)
$$

This assumes that the heat release is periodic, with the same period as the forcing. For laminar flames, this is supported by experimental evidence [42, 43, 47, 48]. This model cannot capture a possible response of the nonlinearity at subharmonics. Also, for laminar flames that oscillate in the absence of forcing at an intrinsic frequency [49], it cannot capture the response that may appear at non-integer multiples of the forcing frequency.

The FDF that is fed into the dispersion relation (4) is then defined as

$$
\operatorname{FDF}(A, i \omega) \equiv \frac{\hat{q}_{1} e^{i \phi_{1}}}{\hat{u}} \overline{\bar{Q}}
$$

where $\hat{u}$ is the Fourier component of the input velocity signal at the burner.

Rather than performing the FDF calculations over all possible frequencies, in the following we provide an argument that allows us to limit the calculations only over certain sets of dangerous frequencies. We first recall that the dispersion relation (4) can be derived from the harmonic balance method [24]. Its solutions, which for a fixed value of the amplitude can be interpreted as the poles of the closed-loop thermoacoustic system, are those for which the loop-gain, $|\mathrm{FDF}||H|$, is equal to 1 and the total (wrapped) phase is equal to 0 . To find limit cycle oscillations, we impose the additional condition that the growth rate is equal to zero. From the loop-gain condition, one can infer that a necessary 
(a)

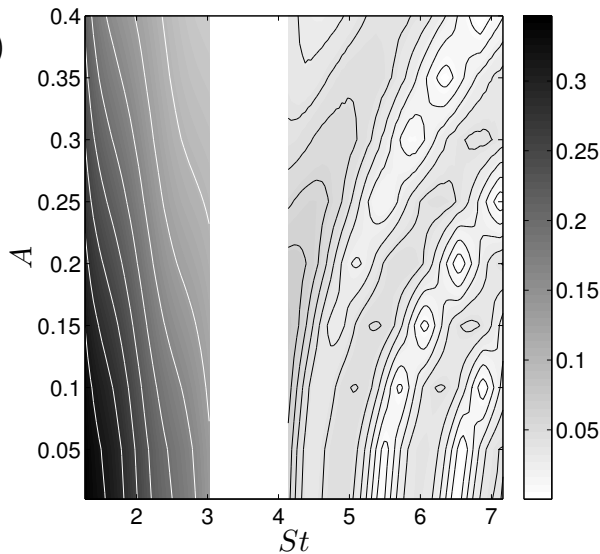

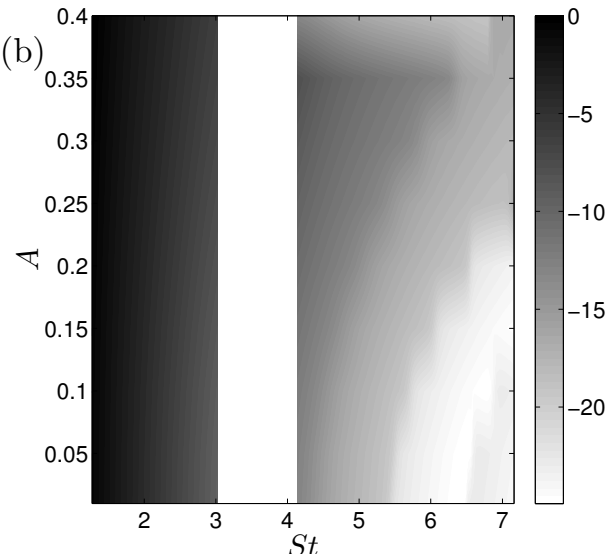

Figure 5: Gain (a) and phase (b) of a laminar, conical flame's FDF. The FDF is evaluated numerically only around frequencies which may give rise to thermoacoustic oscillations.

(but not sufficient) condition for a thermoacoustic oscillation to exist is that either the acoustic transfer function $H$ or the FDF must have a gain larger than 1 . For the acoustic transfer function, this happens close to the acoustic eigenfrequencies, whereas the FDF may or may not have regions in which the gain is larger than 1. If the FDF gain is larger than 1 over some frequency range, the frequency of thermoacoustic oscillations may lie in this region, and can be far from the acoustic eigenfrequencies [50]. It has been shown that these oscillations may persist even in the extreme case in which anechoic boundary conditions for the acoustic network are imposed, and therefore no purely acoustic mode exists $[51,52,53,54]$. For this reason, these thermoacoustic modes have been labelled as intrinsic thermoacoustic modes.

When the unconfined laminar conical flame model we are considering is forced harmonically, its gain $|\mathrm{FDF}|$ never exceeds 1 . Within the $G$-equation framework, this can be proven analytically in the low forcing amplitude limit when curvature corrections on the flame speed are neglected [27, 41, 45]. Numerical and experimental studies show that this holds true even in the fully nonlinear case [14, 35, 48, 55]. For this reason, no intrinsic thermoacoustic instabilities can be observed in our system, and we can deduce that thermoacoustic oscillations are possible only in certain frequency bands, given by the regions in which the acoustic gain $|H|$ is larger than one. For example, at $x_{f}=0.34$ one can see from Fig. 3 that oscillations can be expected only in the $[118,197] \mathrm{Hz}$ and $[303,371] \mathrm{Hz}$ band regions.

This is useful information because we can reduce the cost of the FDF calculations by evaluating the FDF only over these frequency regions ${ }^{2}$. We identify these regions while varying the bifurcation parameter $x_{f}$ over the entire range $\left[\begin{array}{ll}0 & 1\end{array}\right]$. Let us define the Strouhal number $S t \equiv L_{f} f / \bar{U}$, where $L_{f}$ and $\bar{U}$ are the characteristic flame length and

\footnotetext{
${ }^{2}$ A broad knowledge of the FDF is needed to ensure that a flame's gain never exceeds unity. For our model, we already have this information from the literature [14].
} 

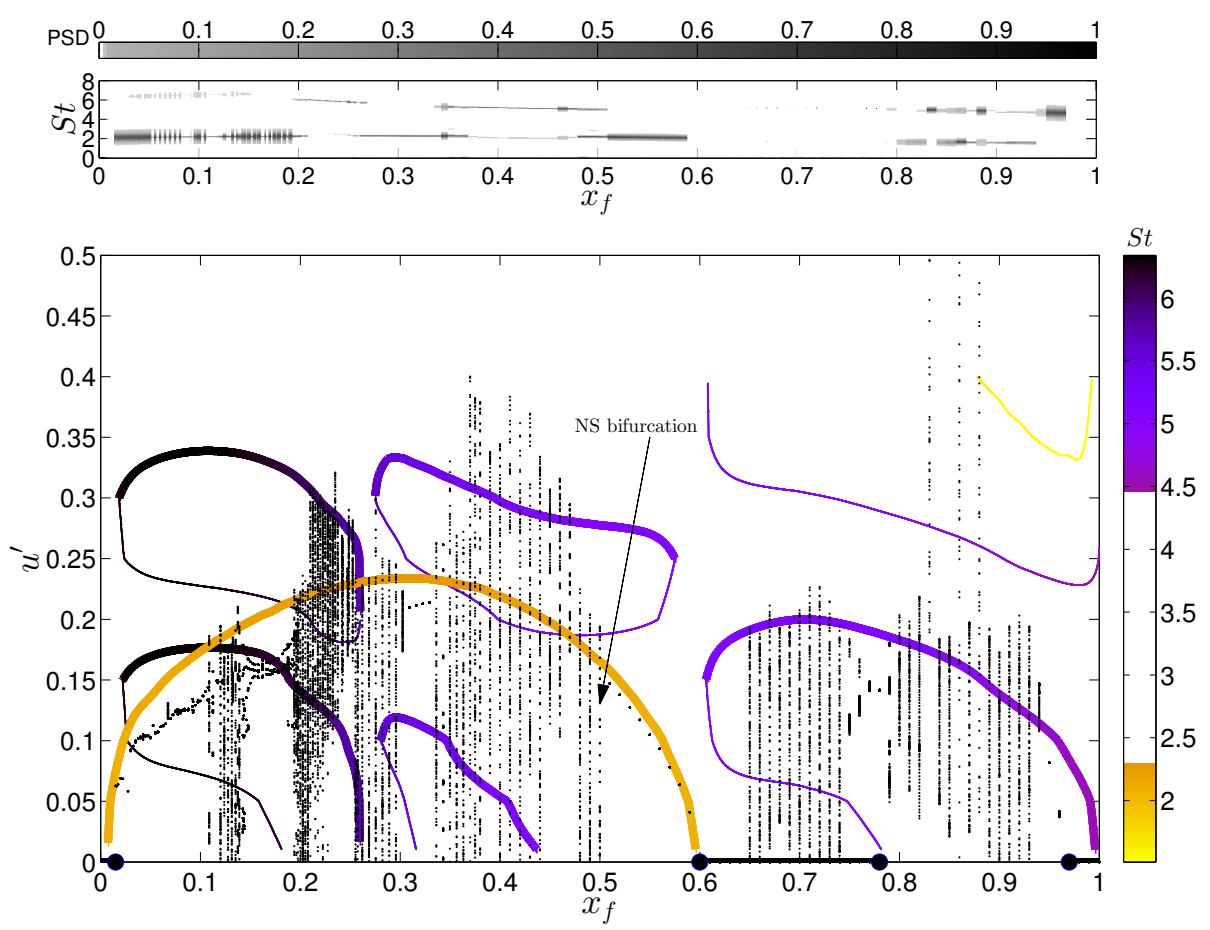

Figure 6: Bottom frame: bifurcation diagrams calculated with time domain simulations (dots) and the FDF method (lines), with the flame position, $x_{f}$, as the bifurcation parameter. Thick and thin lines correspond to stable and unstable limit cycles, respectively, and their colour to the limit cycle oscillation frequency. Dots represent peaks of time domain simulations, as described in [14]. The FDF method predicts Hopf bifurcations and periodic oscillations well (e.g. between $x_{f}=0.50$ and 0.60 ) but cannot predict quasiperiodic oscillations (e.g. between $x_{f}=0.34$ and 0.50 ). The top graph (reproduced from [14] with permission from Cambridge University Press) shows the PSD of time domain simulations at every flame location.

mean flow speed respectively. For the thermoacoustic system under consideration in this study, oscillations are possible only in the frequency ranges $S t \in[1.273,3.024]$, associated with the fundamental acoustic eigenfrequency, and $S t \in[4.138,7.162]$, associated with the second acoustic eigenfrequency. Note that this range is obtained by considering all possible values of $x_{f}$ and is therefore different from the one discussed in the previous paragraph, because the latter was considering only a specific position of the flame. We carry out a detailed evaluation of the FDF in these frequency ranges, varying the amplitude of the oscillation between 0 and 0.4. The FDF gain is shown in Fig. 5, and contains the usual features of conical, premixed flames: the gain is larger at low frequencies and overall it tends to decrease with the amplitude, a signature of the nonlinearity saturation effect. This holds true at low frequencies, whereas at high frequencies the gain can also increase with the amplitude, meaning that subcritical bifurcations and triggering may be observed.

Having calculated both the acoustic transfer function $H$ and the FDF, we can close the thermoacoustic feedback loop as in Fig. 1 and calculate the thermoacoustic eigenfre- 
quencies according to the dispersion relation (4). We recall that (4), deriving from the harmonic balance method, works well when the so-called filtering hypothesis is satisfied, meaning that the closed-loop system does not respond greatly at the harmonics of the input frequency. Experimental and numerical studies have shown that laminar, conical flames act as low-pass filters [41, 43, 55], and Fig. 5 shows that our model contains this feature. Also the acoustics tends to damp high frequencies more, although gain peaks are found at the resonance frequencies. For these reasons, we shall assume that the filtering hypothesis is satisfied.

Limit cycles are found when the dispersion relation (4) is satisfied, with the additional constraint that the growth rate of the oscillations is equal to zero. At this stage, no further approximation has been introduced, because we have knowledge of the FDF at harmonic oscillations. However, to assess the stability of the cycles we need to perturb the saturation amplitude and calculate the shift in frequency and growth rate that it causes, i.e., we want to find the $\Delta s=\Delta \sigma+i \Delta \omega$ that satisfies

$$
\operatorname{FDF}\left(A_{L C}+\Delta A, s_{L C}+\Delta s\right) H\left(s_{L C}+\Delta s\right)-1=0
$$

where $A_{L C}$ and $s_{L C}=i \omega_{L C}$ are a limit cycle solution of (4), and $\Delta A$ is an imposed infinitesimal perturbation. If $\Delta \sigma / \Delta A$ is positive, the limit cycle is unstable, and if it is negative then the cycle is stable. To solve (10) the FDF needs to be extended into the complex plane $\mathbb{C}$. Following [56], we have tried two different techniques: (i) extrusion, by assuming that the FDF does not vary with $\sigma$, and (ii) analytical continuation, by fitting every amplitude slice of the FDF onto a state space. Both methods give the same results.

The bifurcation diagram we obtain by varying the flame position is shown in Fig. 6 . Thick and thin lines indicate stable and unstable limit cycles as predicted by the harmonic balance method. Results are compared with time domain simulations of the same system [14]. Solid lines at $A=0$ indicate regions in which the time domain simulations are linearly stable, and Hopf bifurcations are marked with circles. The dots represent peaks of velocity fluctuations in the time domain. At $x_{f}$ locations where multiple dots are plotted, the oscillations therefore are non-periodic. For example, at $x_{f}=0.5$ time domain oscillations cease to be periodic, and quasiperiodic solutions arise through a Neimark-Sacker bifurcation, marked with an arrow in Fig. 6. The two methods give similar locations of Hopf bifurcations and amplitudes of periodic oscillations. However, the FDF method fails to predict the amplitude of non-periodic oscillations. Further, although many stable limit cycles are predicted by the FDF method, time domain simulations rarely converge to these solutions. This is because they are not, in fact, stable. The FDF criterion for stability misses this because it only considers growth or decay of the mode that is already oscillating. It cannot consider growth or decay of another mode on top of the oscillating limit cycle, which is considered with the FDIDF in the next section.

A similar comparison between the time and frequency domain methods can be found in [14]. However, in that study a mismatch between the locations of the Hopf bifurcations predicted by the FDF and those found by time integration was observed. In this study we have resolved the FDF more accurately in the frequency range in which thermoacoustic oscillations are expected, which has led to a better match between the two methods. 
(a)
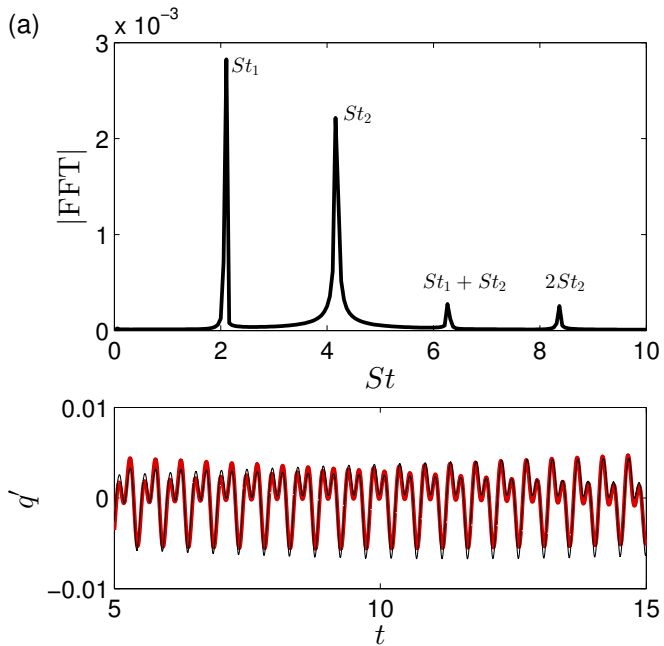

(b)
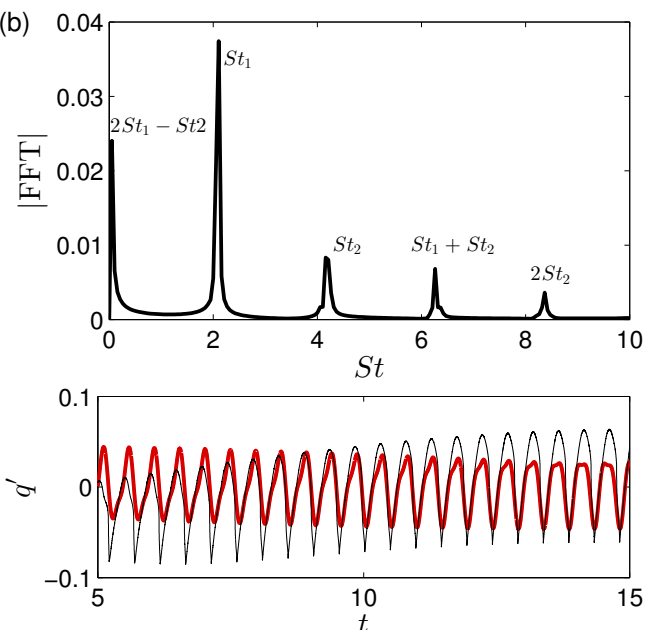

Figure 7: FFT of the heat released by the flame when the forcing is quasiperiodic with the form (11). Top frames: (a) At low forcing amplitudes, $A_{1}=0.01, A_{2}=0.05$, the forcing frequencies dominate the heat release spectrum. (b) At large forcing amplitudes, $A_{1}=0.2, A_{2}=0.25$, peaks at other frequencies become relevant. Bottom frames: nonlinear heat release fluctuations (thin black) and heat release reconstructed using only the peaks at the forcing frequencies (thick red).

\section{FDIDF assumptions and calculation}

By FDIDF, we refer to the frequency domain approximation of the nonlinear flame response to a quasiperiodic velocity perturbation of the form:

$$
u_{a c}^{\prime}=A_{1} \sin \left(\omega_{1} t\right)+A_{2} \sin \left(\omega_{2} t\right)
$$

where $\omega_{1}$ and $\omega_{2}$ are incommensurate frequencies. This choice guarantees that the phase between the two signals does not affect the dynamics. In the following subsections we discuss in details the approximations and assumptions we make concerning the nonlinearity.

\subsection{FDIDF definition}

First, as in the FDF case, we assume that the nonlinearity does not excite the subharmonics of the forcing frequencies, and that no intrinsic dynamical instabilities exist. Because the heat release is a nonlinear function of the forcing signal (11), we expect that its response will contain all the possible combinations of the input frequencies. By using a double Fourier series expansion [24], we can write

$$
q^{\prime}=\sum_{m} \sum_{n} \hat{q}_{m n} \sin \left[\left(m \omega_{1}+n \omega_{2}\right) t+\phi_{m n}\right]
$$

where the heat release amplitude coefficients $\hat{q}_{m n}$ and the phases $\phi_{m n}$ are functions of the input velocity frequencies and amplitudes. The integers $m, n \in \mathbb{Z}$ are varied over all the possible combinations giving a non-negative value of the angular frequency $m \omega_{1}+n \omega_{2}$. 
In order to proceed with the harmonic balance analysis, we need to assume that the heat release response is dominated by the frequency components at the two input frequencies (see Fig. 4), so that it can be approximated by

$$
q^{\prime} \approx \hat{q}_{10} \sin \left(\omega_{1} t+\phi_{10}\right)+\hat{q}_{01} \sin \left(\omega_{2} t+\phi_{01}\right)
$$

This assumption is less well-justified than the filtering hypothesis of the previous section, because the latter only requires that high frequency oscillations will be damped by the system. For the FDIDF, the coupling between the frequencies can also lead to low frequency oscillations (e.g., at an angular frequency of $\left|\omega_{2}-\omega_{1}\right|$ ) for which the filtering hypothesis does not necessarily hold. Therefore, we are implicitly assuming that the nonlinearity's response at these frequencies is either filtered by the system or is weak. This holds true at small forcing amplitude, for which nonlinear effects are small, but it has to be tested at larger amplitudes.

Fig. 7 shows examples on the quality of the FDIDF approximations: at low forcing amplitude (7a) nonlinear effects are weak and the heat release approximated by (13) compares well with the fully nonlinear output. At larger input amplitudes (7b) the quality of the approximation deteriorates. This is because the nonlinearity couples the modes, and high peaks can be observed in the heat release FFT at frequencies which are simple combinations of the input ones. For example, in Fig. $7 \mathrm{~b}$ one can see that the peak at the very low frequency $2 S t_{1}-S t_{2}$ has a large amplitude, meaning that the heat release exhibits large fluctuations over long time scales. The FDIDF approximation cannot see these long time scale fluctuations, as shown in the bottom frame. This is because it ignores all the FFT contributions which are not at $S t_{1}$ and $S t_{2}$. For this reason, we cannot expect the FDIDF method to work well at large amplitudes. Therefore, we limit the FDIDF calculations in amplitude so that both $A_{1}$ and $A_{2}$ are smaller than 0.4 , and their sum is less than 0.5.

The FDIDF is defined as:

$$
\mathrm{FDIDF} \equiv\left[\mathcal{F}_{10}, \mathcal{F}_{01}\right] \equiv \overline{\bar{Q}}\left[\frac{\overline{q_{10} e^{i \phi_{10}}}}{\hat{u}_{10}}, \frac{\hat{q}_{01} e^{i \phi_{01}}}{\hat{u}_{01}}\right]
$$

where $\hat{u}_{10}^{\prime}$ and $\hat{u}_{01}^{\prime}$ are the Fourier components of the input velocity at $\omega_{1}$ and $\omega_{2}$ respectively. $\mathcal{F}_{10}\left(\mathcal{F}_{01}\right)$ contains information on how the amplitude and phase of heat release fluctuations at $\omega_{1}\left(\omega_{2}\right)$ vary when the flame is forced quasiperiodically. The total (non-dimensional) heat release fluctuations are then approximated by

$$
\hat{q}^{\prime} \approx \mathrm{FDIDF} \cdot\left[\hat{u}_{10}, \hat{u}_{01}\right]^{T}=\mathcal{F}_{10} \hat{u}_{10}+\mathcal{F}_{01} \hat{u}_{01}
$$

Note that the heat release in (15) is not a simple linear superposition of two FDFs. This is because the FDIDF's gains and phases are functions of all the four input variables $\left(A_{1}, \omega_{1}, A_{2}, \omega_{2}\right)$. Finally, notice that the FDIDF is a symmetric object with respect to the input pairs $\left(A_{1}, \omega_{1}\right)$ and $\left(A_{2}, \omega_{2}\right)$ so that

$$
\mathcal{F}_{10}\left(A_{1}, \omega_{1}, A_{2}, \omega_{2}\right)=\mathcal{F}_{01}\left(A_{2}, \omega_{2}, A_{1}, \omega_{1}\right)
$$

\subsection{FDIDF amplitude saturation}

In $\S 3$, using knowledge of the flame's gain response from the literature, we performed calculations only for frequencies close to the first two acoustic modes. No information is 


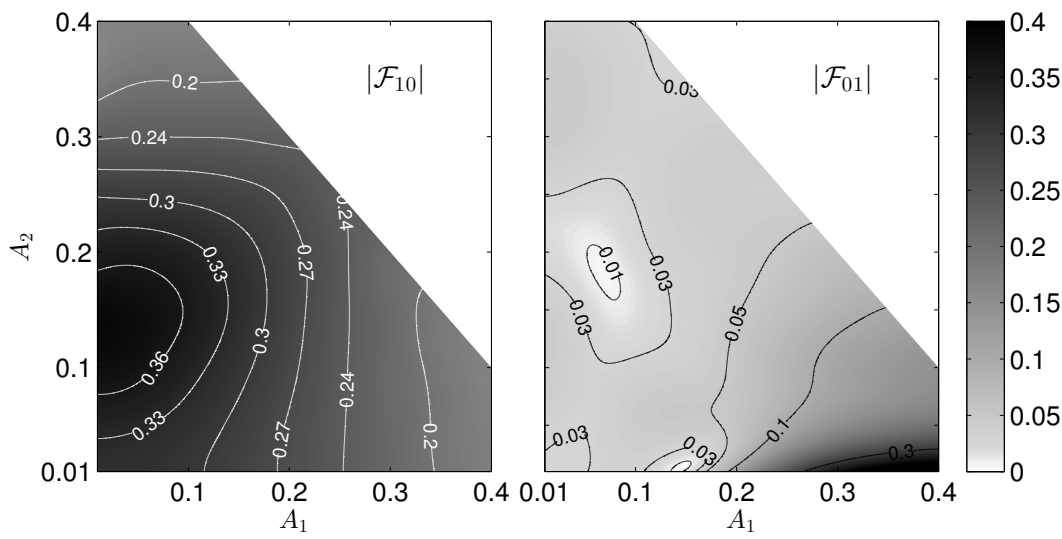

Figure 8: Amplitudes dependence of the FDIDF gains. The forcing frequencies have been fixed at the arbitrary values $S t_{1}=1.513$ and $S t_{2}=5.153$. The region $A_{1}+A_{2}>0.5$ has not been investigated.

available about the gain response of conical flames when they are forced with quasiperiodic signals. However, it is reasonable to assume that, when fixing the amplitude $A_{1}$ and increasing the amplitude $A_{2}$ (or viceversa), the gains of the FDIDF will decrease. This is because we expect the flame nonlinear responses $\mathcal{F}_{10}$ and $\mathcal{F}_{01}$ to saturate, at least on average, with respect to the amplitudes $A_{1}$ and $A_{2}$ of both forcing modes. This is proven to be correct for a simple cubic nonlinearity in [21], where also some experimental evidence of this fact is provided.

We therefore assume that the FDIDF gains are less than 1. As a consequence, we expect that self-excited thermoacoustic oscillations can only be found at frequencies for which the acoustic gain is larger than one (see the FDIDF dispersion relations (18)). These frequency ranges are the same as in the FDF case, because we have not modified the acoustic system. Because we expect two modes to be unstable, it is reasonable to guess that one of the mode's frequencies will be close to the fundamental acoustic frequency, and the other one will be close to the acoustic second acoustic eigenfrequency. Note that, if two modes with similar frequencies were to oscillate simultaneously, beating phenomena could occur, and one should also investigate the coupling between these close frequencies. However, this does not happen for the system we are considering, as was also shown via the time domain analysis carried out in [14] on the same thermoacoustic system. Given this, and the symmetry condition (16), we will limit the calculations to the cases in which the non-dimensional frequencies $S t_{n} \equiv L_{f} f_{n} / \bar{U}$ lie in the ranges $S t_{1} \in[1.273,3.024]$ and $S t_{2} \in[4.138,7.162]$ respectively. In the following, we will refer to mode 1 and mode 2 when referring to oscillations with a frequency in the range spanned by $S t_{1}$ and $S t_{2}$ respectively.

\subsection{FDIDF calculation and validation}

Fig. 8 shows an example of the FDIDF gains as a function of the two forcing amplitudes. The forcing frequencies are fixed at arbitrary values. We observe that the gain of $\mathcal{F}_{10}$ (low frequencies) is generally larger than the gain of $\mathcal{F}_{01}$ (high frequencies); this is in line with the low-pass filter characteristics of the conical flame we are investigating. 


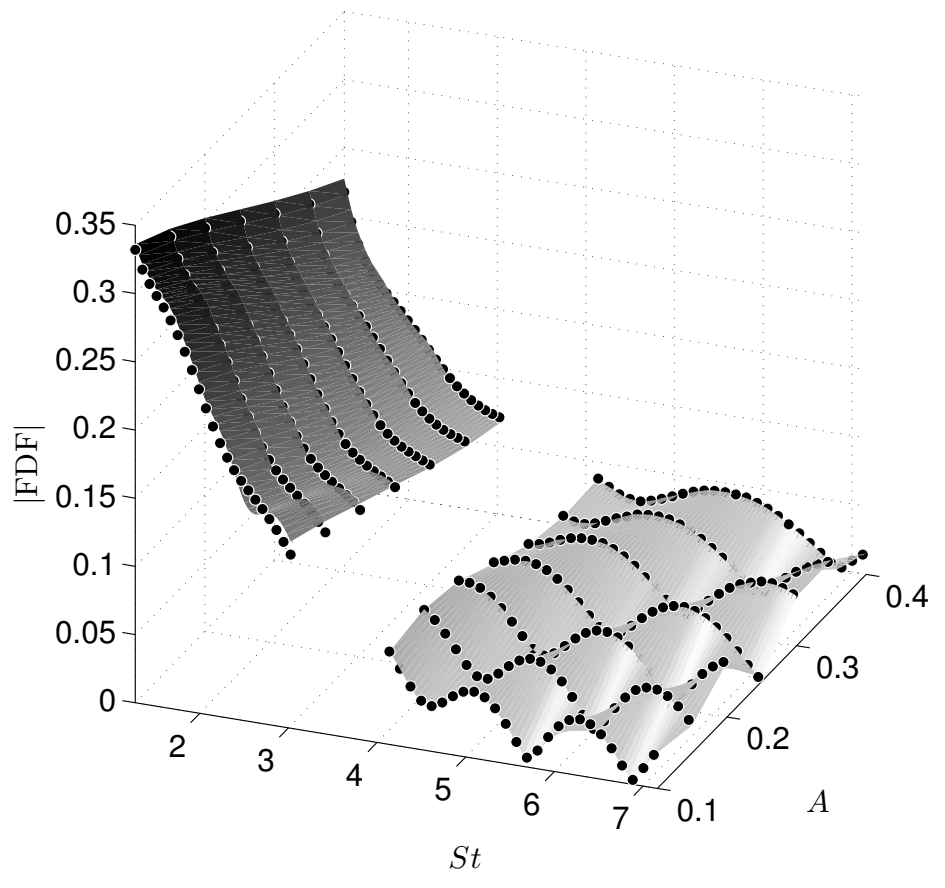

Figure 9: In the limit in which one of the two amplitude vanishes, the FDIDF tends to the FDF. FDIDF limits are plotted as surfaces and the FDF results as dots. In the region $S t \in[1.273$, 3.024] the limit of $\left|\mathcal{F}_{10}\right|$ is plotted fixing $S t_{2}=5.153$ and $A_{2}=0.01$ in (17); in the region $S t \in[4.138,7.162]$ the limit of $\left|\mathcal{F}_{01}\right|$ is plotted fixing $S t_{1}=1.513$ and $A_{1}=0.01$. The results compare well over the entire set of parameters investigated.

Also, for $\mathcal{F}_{10}$ we see that the gain tends to decrease with respect to both amplitudes, as was discussed in the previous section. This is not always true for the $\mathcal{F}_{01}$. It is not surprising because $\mathcal{F}_{01}$ contains the heat release response at frequencies spanned by $S t_{2}$. Even in the FDF analysis we observed that, in this frequency range, the gain does not decrease monotonically with the amplitude, meaning that subcritical Hopf bifurcations and regions with multi-stable solutions may be observed.

In rare cases, we observe that the gain of $\mathcal{F}_{01}$ is larger than one. This always happens when the amplitude of $A_{1}$ is large (between 0.3 and 0.4 ), and the amplitude of $A_{2}$ is at its minimum, 0.01. This is due to the fact that, although we numerically ensure that the two forcing frequencies are incommensurate, their ratio can be close to a simple fraction. For example, in some cases the frequency $S t_{2}$ is close to a harmonic of $S t_{1}$. If the velocity amplitude at $S t_{1}$ is large, the heat release responds significantly also at its harmonics. Because we perform FFTs on signals of finite length, the FDIDF component at $S t_{2}$ will see part of the harmonic contribution of $S t_{1}$, artificially increasing the gain of the second mode (see Fig. 8). This is a source of error which increases when the thermoacoustic eigenfrequencies are close to multiples of each other. It could be reduced by integrating the governing equations over a longer time period, in order to have a better frequency resolution in Fourier space and distinguish the various peak contributions. However, this would lead to an extra numerical cost, which is undesirable. 
A good test to assess the accuracy of the FDIDF calculations is to look at the limit in which the amplitude of one of the two modes goes to zero. From the definitions of the FDIDF and FDF one can verify that:

$$
\begin{array}{ll}
\lim _{A_{2} \rightarrow 0} \mathcal{F}_{10}\left(A_{1}, \omega_{1}, A_{2}, \omega_{2}\right)=\operatorname{FDF}\left(A_{1}, \omega_{1}\right) & \forall \omega_{2} \\
\lim _{A_{1} \rightarrow 0} \mathcal{F}_{01}\left(A_{1}, \omega_{1}, A_{2}, \omega_{2}\right)=\operatorname{FDF}\left(A_{2}, \omega_{2}\right) & \forall \omega_{1}
\end{array}
$$

meaning that $\mathcal{F}_{10}$ tends to the FDF results in the region covered by $S t_{1}$ when $A_{2}$ vanishes and, by exploiting the symmetry condition $(16), \mathcal{F}_{01}$ tends to the FDF results in the region covered by $S t_{2}$ when $A_{1}$ vanishes.

Assuming that the FDIDF is a continuous function, we use the calculations at the smallest amplitudes we have investigated (0.01) as limits. Therefore, the horizontal slice of $\left|\mathcal{F}_{10}\right|$ at $A_{2}=0.01$ and the vertical slice of $\left|\mathcal{F}_{01}\right|$ at $A_{1}=0.01$ in Fig. 8 need to match the FDF gain at $S t=1.513$ and $S t=5.153$ respectively (vertical slices of Fig. 5). Fig. 9 shows this comparison over the entire range of frequencies and amplitudes we have investigated. The limits agree well with the FDF results, with the largest difference between the FDF and the FDIDF limit being about $10^{-3}$.

\section{FDIDF analysis}

We now couple the FDIDF with the acoustic response in a similar fashion as in Fig. 1 and find the dispersion relations that need to be satisfied for quasiperiodic oscillations to exist. The coupling between the acoustic network and the FDIDF is sketched in Fig. 10. Note that, although $\hat{q}_{10}$ is explicitly proportional only to $\hat{u}_{10}$ through $\mathcal{F}_{10}$, the latter is an implicit nonlinear function of both $\hat{u}_{10}$ and $\hat{u}_{01}$. Therefore, the dispersion relations we obtain when imposing the harmonic balance condition are coupled, and need to be simultaneously satisfied:

$$
\begin{aligned}
& \mathcal{F}_{10}\left(A_{1}, s_{1}, A_{2}, s_{2}\right) H_{x_{f}}\left(s_{1}\right)-1=0 \\
& \mathcal{F}_{01}\left(A_{1}, s_{1}, A_{2}, s_{2}\right) H_{x_{f}}\left(s_{2}\right)-1=0
\end{aligned}
$$

Quasiperiodic oscillations of the form (11) exist when the growth rates of the Laplace variables $s_{n}=\sigma_{n}+i \omega_{n}$ are both equal to zero, which is the condition under which the FDIDF was calculated. However, to investigate the stability of the FDIDF solutions, we want to calculate the rate of change of the growth rates when the calculated amplitudes are perturbed. This will yield solutions of (18) with non-zero growth rates. Because we are working with a non-static nonlinearity, the FDIDF is a function of two complex variables, and it is not straightforward to extend it to the complex $\mathbb{C}^{2}$ space. Thus, we decide to use the extrusion method of [56], by assuming that $\operatorname{FDIDF}\left(A_{1}, s_{1}, A_{2}, s_{2}\right)=$ $\operatorname{FDIDF}\left(A_{1}, i \omega_{1}, A_{2}, i \omega_{2}\right)$, which is a zero-order approximation of the FDIDF around the solutions. This complication is not present in the study of [21], because static nonlinearities were used. In that case, the FDIDF is a simpler object and depends only on the forcing amplitudes, not on the frequencies.

\subsection{Linear stability of limit cycles: Neimark-Sacker bifurcations}

A first set of solutions of the FDIDF are those for which the amplitude of one of the two modes is equal to zero. These are the FDF harmonic solutions. For example, if 


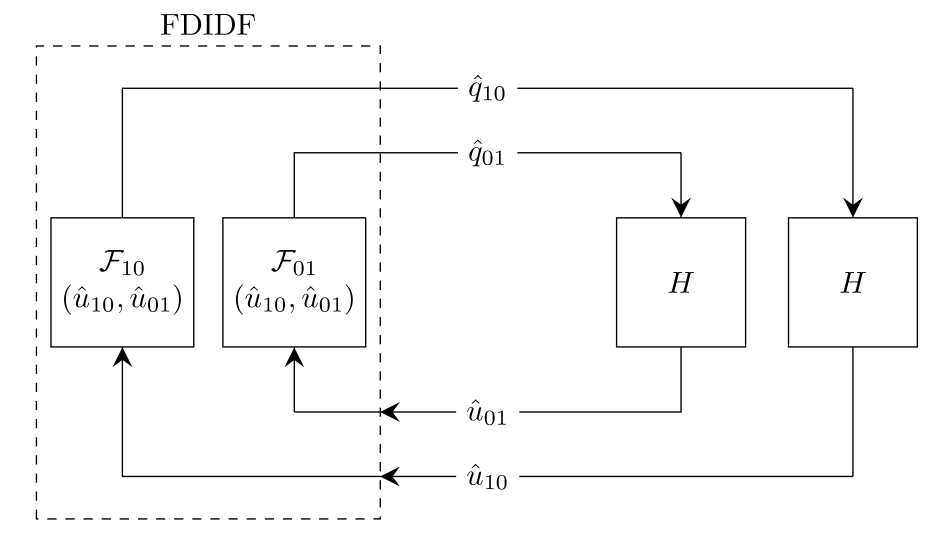

Figure 10: Sketch of the FDIDF feedback loop with the acoustics. The FDIDF (dashed block) is a two-input, two-output nonlinear object. The output is formed with the superposition of two, coupled, nonlinear elements which respond at different frequencies.

The two harmonic components of the quasiperiodic signal are indicated with subscripts 10 and 01 respectively. The implicit dependence of $\mathcal{F}_{10}, \mathcal{F}_{01}$ with respect to both $\hat{u}_{10}$ and $\hat{u}_{01}$ has been highlighted to emphasise that the dispersion relations (18) are coupled.

$A_{2}=0$ then we look for periodic solutions (with zero growth rate) of mode 1. From (18) we have:

$$
\begin{array}{r}
\operatorname{FDF}\left(A_{1}, i \omega_{1}\right) H_{x_{f}}\left(i \omega_{1}\right)-1=0 \\
\mathcal{F}_{01}\left(A_{1}, i \omega_{1}, 0, s_{2}\right) H_{x_{f}}\left(s_{2}\right)-1=0
\end{array}
$$

The first equation derives from the limit (17) and converges to the FDF dispersion relation (4). It is now decoupled from the second equation. We have already calculated its solutions, shown as yellow lines in Fig. 6. The second dispersion relation, however, contains information that the FDF cannot provide. It has to be solved for the frequency $\omega_{2}$ and the growth rate $\sigma_{2}$ by fixing the frequency and amplitude of the other mode at the FDF solution. If the growth rate $\sigma_{2}$ is positive, then oscillations at frequency $\omega_{2}$ are linearly unstable around the limit cycle with amplitude $A_{1}$ and frequency $\omega_{1}$. The onset of these instabilities is known as a secondary Hopf or Neimark-Sacker bifurcation.

Fig. 11 shows the bifurcation diagram of periodic solution when their stability is assessed with the FDIDF method. Most of the limit cycles that were found to be stable with the FDF method are now predicted to be unstable because, according to the solution of (19), oscillations at a different frequency will grow around them. This is consistent with the time integration results, in which we rarely observe periodic oscillations. Time domain and FDIDF results cannot compare perfectly throughout the entire bifurcation map, because the latter neglects contributions away from the input frequencies, which may be important at large amplitudes. However, the FDIDF correctly captures some of the system's bifurcations. For example, analysing Fig. 11 from $x_{f}=0.60$ backwards, time marching results show a supercritical Hopf bifurcation at $x_{f}=0.59$, a NeimarkSacker bifurcation at $x_{f}=0.50$, and an inverse Neimark-Sacker at $x_{f}=0.11$. The 


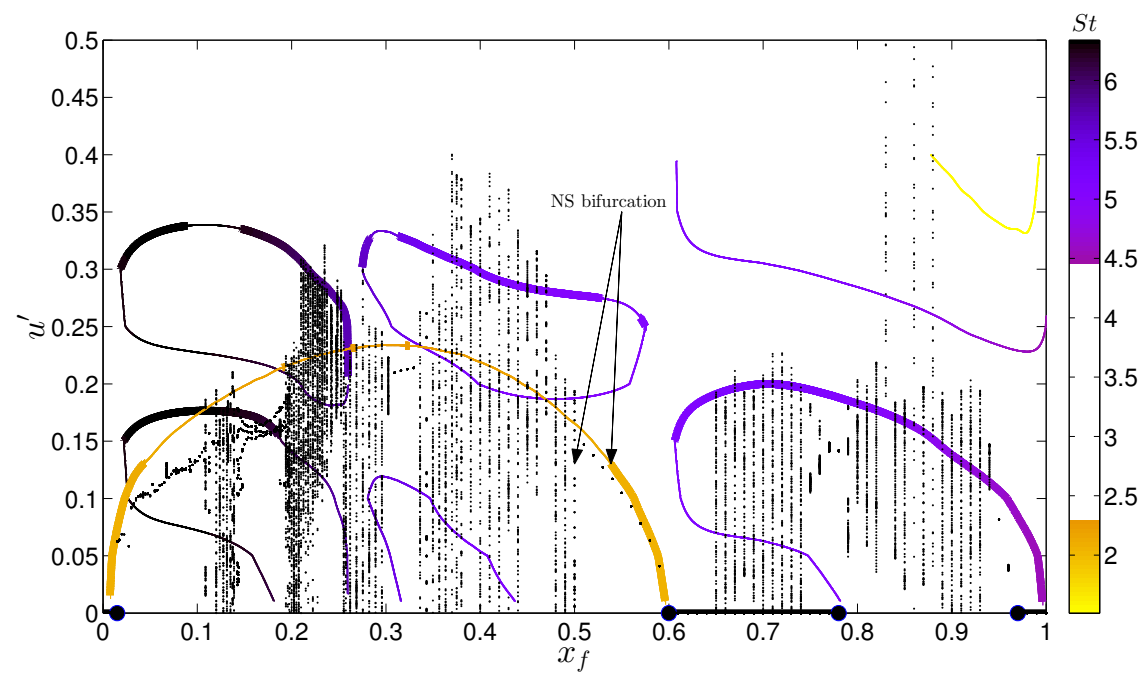

Figure 11: Comparison between time domain (as in Fig. 6) and FDIDF period bifurcation diagrams. The flame position $x_{f}$ is used as a control parameter. Thin and thick lines are used to plot unstable and stable limit cycles respectively. Neimark-Sacker bifurcations are found at the edges of the stable solution with a non-zero amplitude.

FDIDF method locates correctly the first Hopf bifurcation for mode 1, and predicts Neimark-Sacker bifurcations at $x_{f}=0.53$ and $x_{f}=0.045$.

With the FDIDF we can also calculate the frequency of oscillations that grow around limit cycles after Neimark-Sacker bifurcations. At $x_{f}=0.53$ the FDIDF predicts that oscillations with a non-dimensional frequency $S t_{2}=5.0136$ are linearly unstable $\left(\sigma_{2}=2.86 \cdot 10^{-5}\right)$ around the limit cycle with $A_{1}=0.1305$ and $S t_{1}=2.1132$. This prediction can be compared with self-excited time domain results. Fig. 12 shows the FFT of the velocity signal just before and after the Neimark-Sacker bifurcation in the time domain (see Fig. 11). In the former case, the oscillation is dominated by a component at frequency $S t_{1}=2.148$ with intensity $A_{1}=0.1399$. Just after the bifurcation a second high peak appears at $S t_{2}=5.005$. All these results are consistent with the FDIDF predictions.

\subsubsection{Discussion on cost and practical implementation}

The FDIDF is a function of four independent input parameters. As a consequence, the numerical cost of building such an object increases quickly when wide ranges of parameters are investigated. By using the arguments in $§ \S 4.1-4.2$, we limit as much as possible the width of these ranges. However, it is non-trivial to determine how to discretize these regions to appropriately estimate the FDIDF response. Because our model is low-order, we can afford to carry out a very detailed calculation of the FDIDF, and then investigate its dependence on the number of points used. We use about 60 discretization points for each frequency range. We vary the amplitudes in the range $[0.01,0.4]$ in 9 steps, with the additional constraint that their sum does not exceed the threshold value of 0.5 . With these limits, the total number of simulations we run to build the FDIDF is about 200000 . About $70000 \mathrm{CPU}$ hours were required to perform the analysis, which is approximately 5 times more expensive than the continuation method 

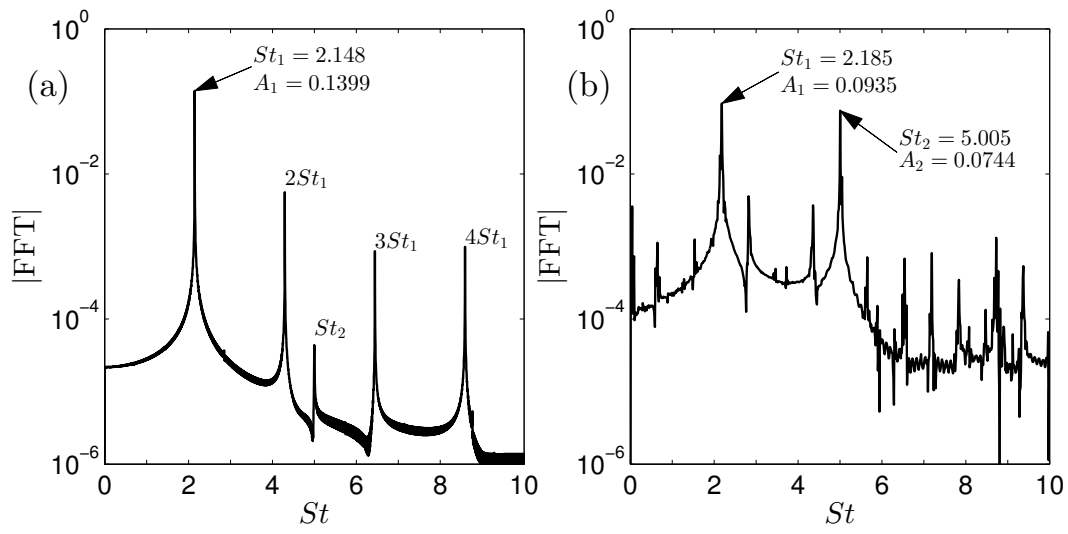

Figure 12: FFT of velocity fluctuations of time domain simulations as described in [14]. (a): $x_{f}=0.51$ the solution is periodic, only a dominant peak at $S t_{1}=2.148$ is found; its harmonics are present but negligible. (b) $x_{f}=0.49$, a second intense peak is found at $S t_{2}=5.005$; the system has undergone a subcritical Neimark-Sacker bifurcation.

used by [12] to calculate limit cycle bifurcations on a similar thermoacoustic system with a continuation algorithm. We then use a four-dimensional cubic spline method to interpolate the real and imaginary parts of the FDIDF between calculated points onto a much finer grid, as functions of the input amplitudes and frequencies. The interpolation is performed both using the full set of simulations or partial information only, to assess the effect of the discretization on the system dynamics.

Fig. 13 shows the relative error of the interpolated FDIDF as a function of the number of points (always uniformly spaced) used for the interpolation. The results at the finest discretization, which are those used for the analysis in the rest of this study, are used as reference. By halving the number of discretization points used for $S t_{1}$ and $S t_{2}$, the cost of the FDIDF is reduced by a factor of 4 and the percentage error is about $5 \%$. However, further reduction in the number of points used for the interpolation lead to larger errors, and significant deviation from the actual dynamic response should be expected. This shows that a large number of calculations is required to accurately estimate the FDIDF. This makes it currently non-affordable for, say, compressible LES studies, in which many CPU hours are already required to calculate the FDF only [57].

Part of the high cost of the current FDIDF analysis is due to the fact that all possible flame positions are investigated. Because a temperature jump follows the flame, the eigenfrequencies vary significantly when $x_{f}$ spans from 0 to 1 , and wide range of frequencies need to be investigated. In practical situations this is probably not the case, and the frequency bands of interest may be narrower, thus reducing the number of calculations required for the FDIDF. Also, we emphasise that, to calculate the stability of limit cycles found with the FDF (as was shown in $\S 5.1$ ), we need only a part of the FDIDF calculation. This is because we examine cases in which one of the two amplitudes is small. The only parameter that has to be varied is the frequency of the small amplitude mode. In this framework, the FDIDF method is much cheaper (it approximately reduces to the cost of two FDFs), and is comparable in cost with the continuation method described 


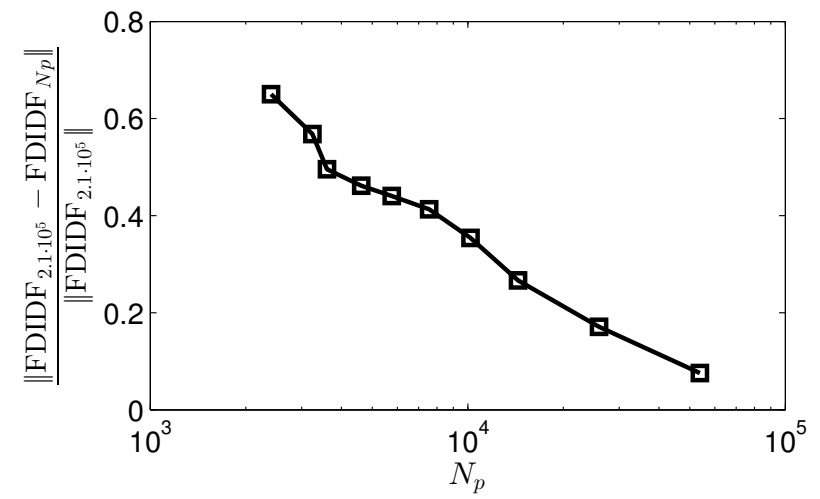

Figure 13: Interpolated FDIDF relative error dependence with respect to the number of discretization points $N_{p}$ used. Choosing fewer than 30000 points leads to deviations from the actual response larger than $10 \%$.

by [12]. The latter remains more accurate, because it studies the stability of periodic solutions (i.e., the spectrum of the oscillations may contain peaks at the harmonics of the fundamental frequency), whereas the FDIDF is limited to harmonic solutions (i.e., the spectrum of the oscillations contains only one peak at the fundamental frequency). The advantage of the FDIDF is that it can be reused in different acoustic networks to calculate the stability of several thermoacoustic systems.

The use of the FDIDF to assess the stability of periodic solutions could also be exploited in experiments at approximately the cost of two FDFs by means of the following procedure: (i) measure an FDF; (ii) obtain harmonic solutions and their stability - with respect to a single mode - with the harmonic balance; (iii) for solutions that are predicted to be stable by the FDF method, perform another set of experiments to assess again their stability with respect to other forcing frequencies. This is accomplished by forcing the flame with a signal of the form (11), by fixing the amplitude and frequency of a mode at the FDF solution and the amplitude of the other mode at a small value. The only parameter left is the frequency of the second mode. It has to be varied over a range of dangerous frequencies, which can be obtained by the FDF results and the acoustic response. The stability of the FDF solutions with respect to other frequencies can then be calculated following the procedure described in $\S 5.1$.

\subsection{Prediction and stability of quasiperiodic oscillations}

Once limit cycles have become unstable, thermoacoustic oscillations converge towards another stable solution. This can be another periodic solution, with a different frequency and amplitude, a quasiperiodic attractor, or even a strange attractor. The FDIDF can approximate the location and stability of periodic and quasiperiodic solutions, but cannot predict the existence of other types of attractors, which were shown to exist in this type of thermoacoustic system by $[11,14]$.

When looking for quasiperiodic attractors, the dispersion relations (18) need to be solved by fixing the growth rates $\sigma_{1}$ and $\sigma_{2}$ at zero, and looking for solutions with finite amplitudes for both modes. We rely on numerical techniques to find the roots of (18) that satisfy this conditions starting from a good initial guess. Because five parameters 
(two amplitudes, two frequencies and the bifurcation parameter) can be varied, a large number of initial guesses can be chosen, which is numerically inefficient. To reduce the numerical cost, we first locate isolated solutions by starting from a coarse grid of initial guesses that covers the parameter space. Then, we extend the solutions to continuous lines with an arclength continuation method by slowly varying the bifurcation parameter.

\subsubsection{Stability criterion}

We find several sets of quasiperiodic solutions and require a criterion to assess their stability. From a dynamical system viewpoint, the coupled evolution of the oscillations' amplitudes can be written in terms of a linear operator $L$ and a nonlinear operator $N(\boldsymbol{A})$ as

$$
\frac{\mathrm{d} A_{j}}{\mathrm{~d} t}=L_{j} A_{j}+N_{j}(\boldsymbol{A}) \equiv \sigma_{j}(\boldsymbol{A}) A_{j}
$$

where $\boldsymbol{A}$ is the amplitudes vector. Although the explicit expressions for the linear and nonlinear operators are not known, $\sigma_{j}$ represents a nonlinear growth rate, in the sense that its intensity varies with the amplitudes of the oscillations. When at least one $\sigma_{j}$ equals zero, a non-trivial solution (with a finite amplitude) to the dynamical system has been found. The amplitude of each mode varies with respect to the value of its growth rate only, which is implicitly a function of all the amplitudes. For our system, which contains only two modes, the dynamical system (20) reduces to:

$$
\begin{aligned}
& \dot{A}_{1}=\sigma_{1}\left(A_{1}, A_{2}\right) A_{1} \\
& \dot{A}_{2}=\sigma_{2}\left(A_{1}, A_{2}\right) A_{2}
\end{aligned}
$$

Equations (21) will slowly vary the oscillations' amplitudes, which in turn will change the growth rates and frequencies according to the solution of (18) at the current amplitudes. Eqs. (21) were also discussed in [21], where their interpretation in terms of an averaging procedure was also provided.

Let us now indicate a solution of (21) with overlines. These solutions are fixed point if both amplitudes are equal to zero, limit cycles if only one amplitude is zero, or quasiperiodic if both amplitudes are non-zero. By linearising eq.(21) around a solution the evolution of small perturbations, indicated with $\Delta$, is given by:

$$
\frac{d}{d t}\left[\begin{array}{c}
\Delta A_{1} \\
\Delta A_{2}
\end{array}\right]=\left[\begin{array}{cc}
\frac{\partial \sigma_{1}}{\partial A_{1}} \bar{A}_{1}+\bar{\sigma}_{1} & \frac{\partial \sigma_{1}}{\partial A_{2}} \bar{A}_{1} \\
\frac{\partial \sigma_{2}}{\partial A_{1}} \bar{A}_{2} & \frac{\partial \sigma_{2}}{\partial A_{2}} \bar{A}_{2}+\bar{\sigma}_{2}
\end{array}\right]\left[\begin{array}{c}
\Delta A_{1} \\
\Delta A_{2}
\end{array}\right] \equiv \boldsymbol{J} \boldsymbol{\Delta} \boldsymbol{A}
$$

where the partial derivatives are evaluated at the solution. If the eigenvalues of the Jacobian $\boldsymbol{J}$ have negative real parts, the solution under consideration is stable.

It is worth discussing the forms that the Jacobian assumes for the different types of solutions. For a fixed point, both amplitudes vanish and $\boldsymbol{J}$ simply contains the growth rates $\sigma_{1}$ and $\sigma_{2}$ on the main diagonal, retrieving the classic linear stability result. For a limit cycle solution (say of mode 1), the Jacobian takes the form

$$
\boldsymbol{J}_{L C}=\left[\begin{array}{cc}
\frac{\partial \sigma_{1}}{\partial A_{1}} \bar{A}_{1} & \frac{\partial \sigma_{1}}{\partial A_{2}} \bar{A}_{1} \\
0 & \bar{\sigma}_{2}
\end{array}\right]
$$

and has eigenvalues $\partial \sigma_{1} / \partial A_{1} \bar{A}_{1}$ and $\bar{\sigma}_{2}$. Because $\bar{A}_{1}$ is positive, the stability is determined by the sign of $\partial \sigma_{1} / \partial A_{1}$ (the FDF condition) and $\sigma_{2}$. This corresponds to the 
stability condition that was intuitively discussed in the previous section. Furthermore, the eigenvector corresponding to the limit cycle eigenvalue $\partial \sigma_{1} / \partial A_{1} \bar{A}_{1}$ is orientated along the $A_{1}$ direction. The second eigenvector, however, has a non-trivial direction and can be calculated only having the FDIDF. We will shortly return to the significance of these eigenvectors in the FDIDF analysis.

Lastly, for quasiperiodic solutions we obtain that the stability is determined by the eigenvalues of the Jacobian

$$
\boldsymbol{J}_{Q P}=\left[\begin{array}{ll}
\frac{\partial \sigma_{1}}{\partial A_{1}} \bar{A}_{1} & \frac{\partial \sigma_{1}}{\partial A_{2}} \bar{A}_{1} \\
\frac{\partial \sigma_{2}}{\partial A_{1}} \bar{A}_{2} & \frac{\partial \sigma_{2}}{\partial A_{2}} \bar{A}_{2}
\end{array}\right]
$$

544 This is not exactly the condition that was suggested by [21], whose Jacobian does not depend on the solution amplitudes. Nonetheless, condition (24) derives from the linearisation of the amplitudes' evolution around a solution. Given that we retrieve correct physical conditions for the stability of fixed point and limit cycles, we shall expect it to hold even for quasiperiodic oscillations.

Two methods can be used to calculate the partial derivatives of the growth rates with respect to the amplitudes. By brute force, in analogy with eq. (10), one can fix one amplitude at its solution's value, slightly perturb the other amplitude, and determine the variations in frequency and growth rate of both modes by solving (18) with an iterative method. Alternatively, the implicit function theorem may be used, as suggested by [21]. The latter is quicker and more reliable because no iterative methods need to be used. Details on the implicit function theorem method are given in Appendix A. Both methods have been tested and yield the same results.

\subsubsection{FDIDF bifurcation analysis}

Fig. 14 contains the FDIDF solutions when the bifurcation parameter is varied between $0.40 \leq x_{f} \leq 0.60$. Limit cycle solutions lie on $A_{1}=0$ and $A_{2}=0$, whereas solutions for which both amplitudes are non-zero are quasiperiodic. We have plotted with black filled circles attractors (solutions for which both eigenvalues have a negative real part), with red empty circles repellors (both eigenvalues have a positive real part), and with red squares saddle-nodes (one eigenvalue has a positive real part, and the other a negative real part). The latter are particularly interesting because thermoacoustic oscillations can be first attracted towards them along their stable manifold, and only later diverge along the unstable manifold towards an attractor. If the growth rate of the unstable mode is small, the oscillations may persist for a long time around the saddle-node state. This can be problematic for time domain simulations or experiments, because the system has to be observed for a long time before being sure that the final attractor has been reached. Saddle-nodes in thermoacoustic systems were also discussed in [11], where they were referred to as "unstable attractors".

A convenient way of representing the FDIDF results is through phase-planes. A phase-plane contains the trajectories that the amplitudes will follow before converging to an attractor. Starting from different initial conditions can lead thermoacoustic oscillations towards different attractors. The set of initial conditions that converge towards an attractor is known as the basin of attraction of the attractor. On a theoretical basis, it should be possible to identify the basins of attraction boundaries by investigating the growth rates of the thermoacoustic modes while varying the oscillations' amplitudes. 

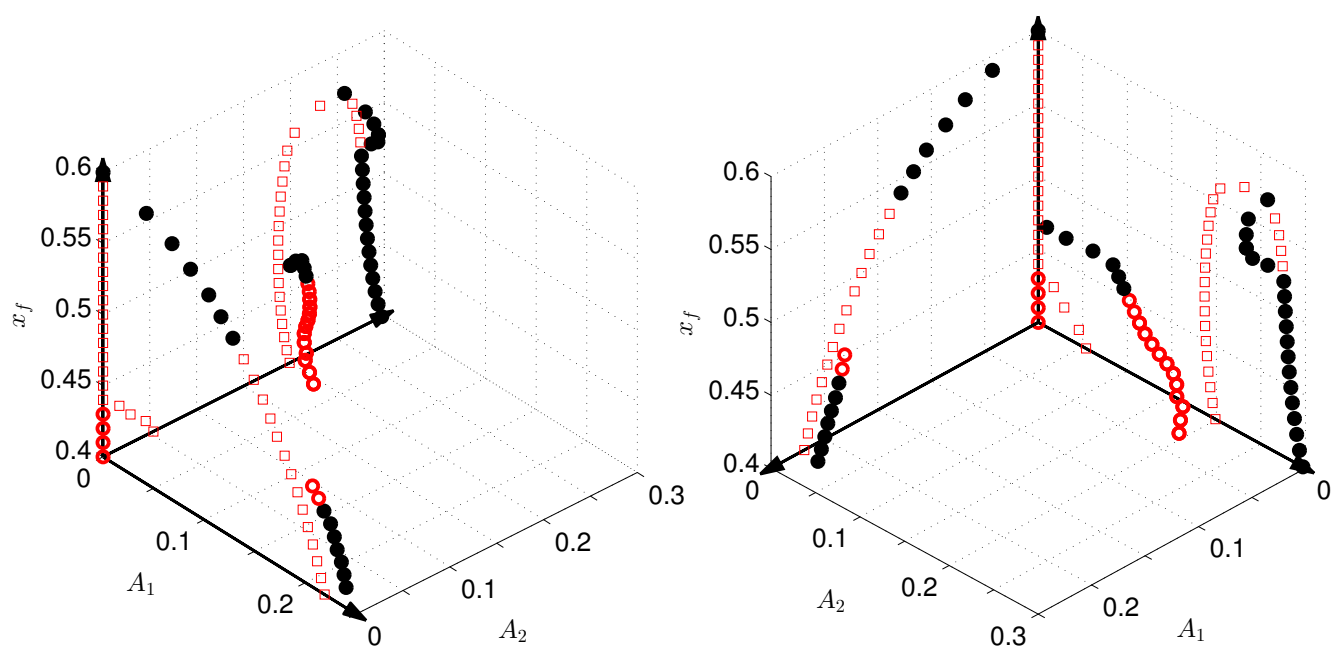

Figure 14: FDIDF bifurcation diagram in the region $0.40 \leq x_{f} \leq 0.60$. The FDF solutions of mode 1 and 2 lie on the $A_{2}=0$ and $A_{1}=0$ planes, respectively. The fixed point solutions lie on the line $A_{1}=A_{2}=0$. Super- and subcritical quasiperiodic oscillations are found. The stability of all solutions is assessed with the FDIDF conditions. Stable attractors are indicated with filled black circles, repellors with empty red circles and saddle-nodes with empty red squares. Two views of the same bifurcation diagram are shown.

However we find that when we are not close to solutions of our system, the growth rates quickly become large. The FDIDF was not evaluated under these conditions, therefore it cannot be used to build the phase-planes because the extrusion method we adopted is no longer valid. Note that, for a static nonlinearity as the one considered by [21], this problem does not arise because the FDIDF is a function of the amplitudes only.

For the non-static nonlinearity we are considering in this study, the FDIDF can still be used to estimate the phase-planes. This is accomplished by calculating the eigenvectors of the Jacobian (22). By means of the Centre Manifold Theorem [58], the eigenspaces spanned by the eigenvectors associated with the stable and unstable eigenvalues are locally tangent to the stable and unstable manifolds respectively. A sketch of the phaseplanes of our system across the Neimark-Sacker bifurcation at $x_{f}=0.53$ is shown in Fig. 15. Stable and unstable solutions are plotted with the same shape and colour scheme of Fig. 14, together with vectors pointing in the direction of their eigenvectors. For saddle-nodes, these vectors are locally tangent to the stable and unstable manifolds. For attractors and repellors, the eigenvalues and eigenvectors of $\boldsymbol{J}$ can be complex-valued. In this case, trajectories will spiral inwards/outwards the solution. We have also sketched with dashed lines possible heteroclinic orbits. A heteroclinic orbit is a path that connects an unstable solution to a stable one. Note that some solutions may be missing from our maps, because they can lie in a region we have not investigated (large amplitudes or amplitudes smaller than 0.01), or they can be strange attractors that we cannot locate.

Although we have only partial information about phase-planes, they help to identify possible routes that thermoacoustic oscillations undertake before converging to an attractor. For example, let us consider Fig. $15 \mathrm{a}$, which corresponds to the $x_{f}$ location just before the Neimark-Sacker bifurcation marked in Fig. 11. Starting from the quies- 

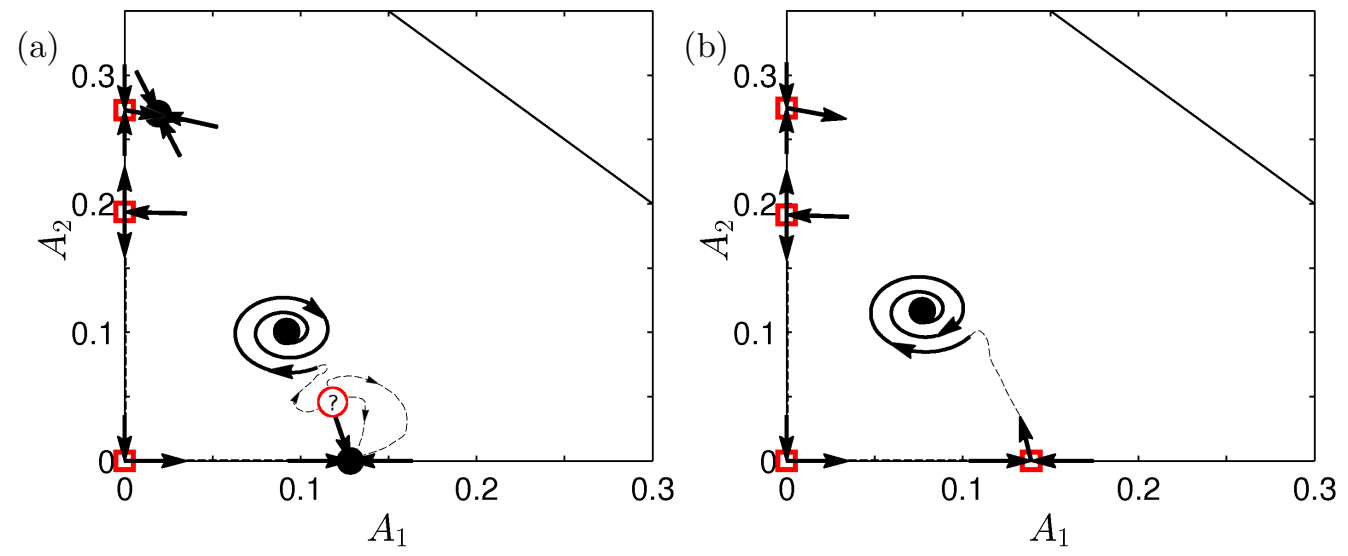

Figure 15: Sketch of phase-planes before (a) and after (b) the Neimark-Sacker bifurcation at $x_{f}=$ 0.53. The arrows pointing inwards and outwards the solutions indicate the directions of the Jacobian eigenvectors with a negative and positive growth rate, respectively. Some hypothetical heteroclinic orbits are sketched with dashed lines. Across the bifurcation, the amplitude $A_{1}\left(A_{2}\right)$ of the attractor reached starting from a quiescent state suddenly decreases (increases).

cent state $A_{1}=A_{2}=0$, the oscillations will be attracted towards the stable limit cycle solution along $A_{2}=0$. However, starting from an excited state with $A_{2} \neq 0$ or by triggering the system, the oscillations may converge to a different attractor. Here, the other possible attractors are quasiperiodic. We have added a non-calculated solution (marked with a question mark) in order to sketch some heteroclinic paths. The position of this solution however is not entirely arbitrary; by slowly varying the bifurcation parameter, the limit cycle solution at $A_{2}=0$ loses its stability. A possible scenario is that, at the bifurcation point, a quasiperiodic repellor (or another type of oscillation) collapses onto the stable limit cycle solution. After the bifurcation (Fig. 15b), the limit cycle on $A_{1}$ turns into a saddle-node, changing the topology of the phase-plane. Now, starting from a quiescent state thermoacoustic oscillations are first attracted towards the limit cycle solution along the $A_{2}=0$ axis (which is the limit cycle stable manifold), and only later are repelled from it along the unstable manifold towards the stable quasiperiodic oscillation. This is exactly what is observed in time domain simulations, although not shown here. Analogous time domain results can be find in [11].

Lastly, Fig. 15 also shows that the amplitudes $A_{1}$ and $A_{2}$ suddenly vary across the bifurcation. This is possible across a Neimark-Sacker bifurcation, as solutions are suddenly attracted towards a different attractor. Time domain results of the same bifurcation shown in Fig. 12 are in line with this FDIDF prediction. Indeed, The FFT of the time signal before and after the bifurcation shows that the amplitudes $A_{1}\left(A_{2}\right)$ suddenly decreases (increases) across the bifurcation. This feature of Neimark-Sacker bifurcations is also seen in the time domain results shown in Fig. 11. At $x_{f}=0.50$ the maximum amplitude of the oscillations suddenly deviates from the limit cycle amplitude before the bifurcation. A fair comparison between the oscillation amplitudes predicted by the FDIDF and time marching is seen by looking at the position of the stable quasiperiodic 


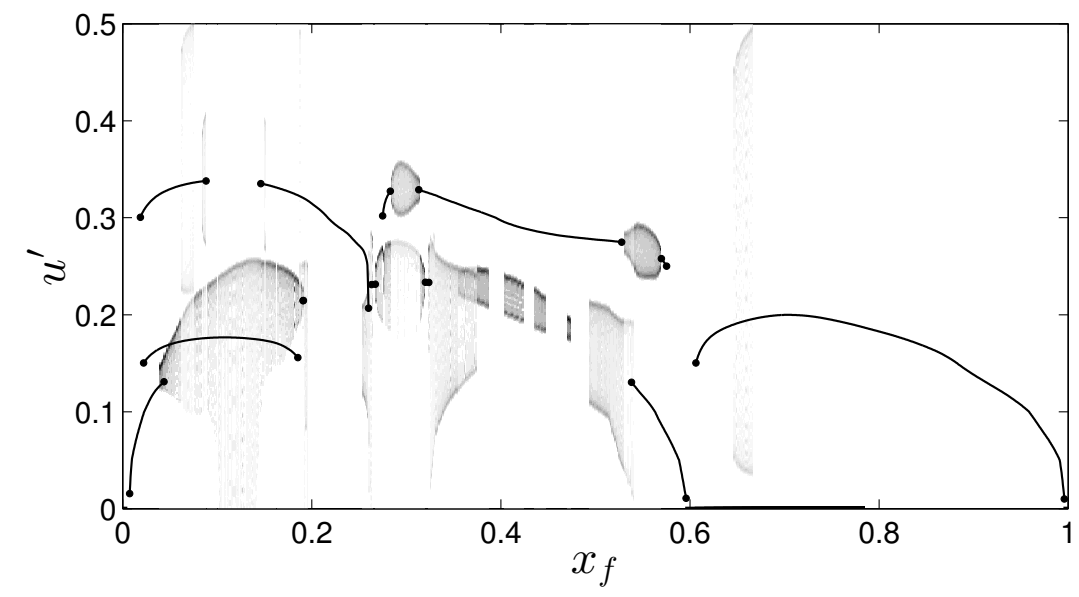

Figure 16: Overview of the FDIDF bifurcation diagram. PDF of stable quasiperiodic solutions' peaks (shaded regions) are plotted on top of stable limit cycle amplitudes (lines). The PDF intensity is higher in darker regions. The location of Neimark-Sacker bifurcations is highlighted with dots.

attractor in Fig. 15 and the intensity of the peaks in Fig. 12. Note that, however, these figures contain information at slightly different values of $x_{f}$, because the location of the Neimark-Sacker bifurcation predicted by the two methods is slightly different, due to the FDIDF approximations.

We conclude this study by showing in Fig. 16 the bifurcation diagram calculated with the FDIDF in the entire range $0 \leq x_{f} \leq 1$. We have plotted only the peaks of stable oscillations, which are those observable in self-excited experiments or time domain simulations. For limit cycle solutions, these peaks are shown as lines corresponding at the oscillations amplitude. For quasiperiodic solutions, we calculate the Probability Density Functions (PDF) of their peaks. These are shown as shaded regions in Fig. 16. The locations of Neimark-Sacker bifurcations have been highlighted with black dots. One can see that there is a nice match between their location and the onset of stable quasiperiodic solutions. This does not happen if we apply the stability criterion for quasiperiodic solutions contained in [21]. In the region $0.60 \leq x_{f} \leq 1$, quasiperiodic oscillations tend to have a large amplitude, which exceeds the $A=0.5$ threshold we have set when calculating the FDIDF. This is partly consistent with time domain results, in which very large oscillations, e.g. at $x_{f}=0.83$, are occasionally observed. In some regions, multiple stable solutions are found. With time marching methods, a thorough investigation of the initial condition is required to find these solutions.

\section{Summary and conclusions}

We have presented a numerical approach for the investigation of non-periodic thermoacoustic oscillations. A Flame Double Input Describing Function (FDIDF) of a nonstatic nonlinear flame model based on the $G$-equation has been calculated by forcing the flame with a quasiperiodic signal composed of two harmonic components with independent amplitudes and incommensurate frequencies. The FDIDF assumptions and 
limitations have been outlined, and it has been tested against the Flame Describing Function (FDF) in the limit in which the amplitude of a mode is small. The FDIDF has been embedded into a thermoacoustic network and, through the harmonic balance method, stable and unstable thermoacoustic oscillations have been calculated. Furthermore, a criterion to assess their stability has been derived.

The FDIDF contains a far more accurate approximation of the nonlinear flame response than the FDF. Exploiting all its information, one can predict the amplitude and stability of quasiperiodic solutions. Also, via the Centre Manifold Theorem, it can be used to sketch phase-planes to understand the path that thermoacoustic oscillations trajectories will take. Quantitative comparisons between the FDIDF and time marching results have been presented. We have shown that the FDIDF is capable of predicting the location of Neimark-Sacker bifurcations, the frequency of the unstable modes and the amplitude of the final quasiperiodic oscillations. We have discussed in detail the change in behaviour of a system at a Neimark-Sacker bifurcation, across which a new mode becomes unstable and the amplitude of the oscillations varies abruptly. This can lead to quasiperiodic oscillations or mode-switching to another stable periodic oscillation at a different frequency. Neither type of behaviour can be predicted by linear stability analysis nor by the FDF framework.

Although the FDIDF is an expensive object to calculate, for simple dynamical flame models, such as the $G$-equation, this is affordable. Also, we have shown how its cost can be greatly reduced if one is interested in calculating only the stability of limit cycles. This accounts for the nonlinear interaction between modes, which the FDF ignores, and provides the location of Neimark-Sacker bifurcations. Only the information at which one of the amplitudes is fixed at a very small value is needed for this, and the cost of the FDIDF reduces to the cost of a second FDF, making it affordable for experimental purposes too. We find that, for our system, most of the limit cycles that are predicted to be stable by the FDF method, are predicted to be unstable by the FDIDF method. This is consistent with self-excited time marching results of the same thermoacoustic system. Within this framework, the FDIDF is capable of predicting the frequency of oscillations that will grow in time around limit cycles. Knowing these frequencies, Helmholtz resonators can be tuned and retro-fitted to the thermoacoustic system in order to make it less prone to oscillations.

\section{Acknowledgements}

We thank Prof. Jonas Moeck for useful discussions and comments on this study. This project was funded by the European Research Council through Project ALORS 2590620.

\section{Appendix A. Growth rate variations by implicit function theorem}

For convenience, let us rewrite the dispersion relations (18) in a compact form by splitting them into real and imaginary part as $\boldsymbol{N}(\boldsymbol{A}, \boldsymbol{y}) \equiv\left(N_{10}^{\mathrm{Re}}, N_{10}^{\mathrm{Im}}, N_{01}^{\mathrm{Re}}, N_{01}^{\mathrm{Im}}\right)=\mathbf{0}$, 
where we have defined

$$
\begin{aligned}
& N_{10}^{\operatorname{Re}} \equiv \operatorname{Re}\left[\mathcal{F}_{10}\left(A_{1}, \omega_{1}, A_{2}, \omega_{2},\right) H\left(\sigma_{1}+i \omega_{1}\right)-1\right] \\
& N_{10}^{\operatorname{Im}} \equiv \operatorname{Im}\left[\mathcal{F}_{10}\left(A_{1}, \omega_{1}, A_{2}, \omega_{2},\right) H\left(\sigma_{1}+i \omega_{1}\right)-1\right] \\
& N_{01}^{\operatorname{Re}} \equiv \operatorname{Re}\left[\mathcal{F}_{01}\left(A_{1}, \omega_{1}, A_{2}, \omega_{2},\right) H\left(\sigma_{2}+i \omega_{2}\right)-1\right] \\
& N_{01}^{\operatorname{Im}} \equiv \operatorname{Im}\left[\mathcal{F}_{01}\left(A_{1}, \omega_{1}, A_{2}, \omega_{2},\right) H\left(\sigma_{2}+i \omega_{2}\right)-1\right]
\end{aligned}
$$

where $\boldsymbol{A} \equiv\left(A_{1}, A_{2}\right)$ is the vector of amplitudes, and $\boldsymbol{y} \equiv\left(\sigma_{1}, \omega_{1}, \sigma_{2}, \omega_{2}\right)$ is the vector of growth rates and frequencies. This is a system of four equations through which the four dependent variables (frequencies and growth rates) are implicit functions of the amplitude levels, i.e., $\boldsymbol{y}=\boldsymbol{y}(\boldsymbol{A})$. By implicit differentiation of the dispersion relations, one obtains

$$
\mathrm{d} \boldsymbol{N}=\frac{\partial \boldsymbol{N}}{\partial \boldsymbol{y}} \mathrm{d} \boldsymbol{y}+\frac{\partial \boldsymbol{N}}{\partial \boldsymbol{A}} \mathrm{d} \boldsymbol{A}=\mathbf{0}
$$

or, by rearranging

$$
\frac{\mathrm{d} \boldsymbol{y}}{\mathrm{d} \boldsymbol{A}}=\left[\begin{array}{ll}
\frac{\partial \sigma_{1}}{\partial A_{1}} & \frac{\partial \sigma_{1}}{\partial A_{2}} \\
\frac{\partial \omega_{1}}{\partial A_{1}} & \frac{\partial \omega_{1}}{\partial A_{2}} \\
\frac{\partial \sigma_{2}}{\partial A_{1}} & \frac{\partial \sigma_{2}}{\partial A_{2}} \\
\frac{\partial \omega_{2}}{\partial A_{1}} & \frac{\partial \omega_{2}}{\partial A_{2}}
\end{array}\right]=-\left(\frac{\partial \boldsymbol{N}}{\partial \boldsymbol{y}}\right)^{-1} \frac{\partial \boldsymbol{N}}{\partial \boldsymbol{A}}
$$

The latter expression yields the growth rates and frequencies sensitivities with respect to amplitudes variations. The right hand side terms can be evaluated by finite difference by imposing small perturbations (one by one) in the dispersion relations (A.1). No iterative methods are required when using the implicit function theorem, which makes the method more reliable because is not susceptible to convergence problems.

\section{References}

[1] W. Lang, T. Poinsot, S. Candel, Active control of combustion instability, Combustion and Flame 70 (1987) 281-289.

[2] M. A. Heckl, Active control of the noise from a Rijke tube, Journal of Sound and Vibration 124 (1) (1988) 117-133.

[3] A. P. Dowling, The calculation of thermoacoustic oscillations, Journal of Sound and Vibration 180 (4) (1995) 557-581.

[4] S. R. Stow, A. P. Dowling, Thermoacoustic oscillations in an annular combustor, in: Proceedings of ASME Turbo Expo 2001, 2001, pp. GT2001-0037.

[5] F. E. C. Culick, Unsteady motions in combustion chambers for propulsion systems, AGARDograph, RTO AG-AVT-039, 2006.

[6] N. Noiray, D. Durox, T. Schuller, S. Candel, A unified framework for nonlinear combustion instability analysis based on the Flame Describing Function, Journal of Fluid Mechanics 615 (2008) $139-167$.

[7] B. Schuermans, Modeling and control of thermoacoustic instabilities, Ph.D. thesis (2003).

[8] M. R. Bothien, J. P. Moeck, A. Lacarelle, C. O. Paschereit, Time domain modelling and stability analysis of complex thermoacoustic systems, Proceedings of the Institution of Mechanical Engineers, Part A: Journal of Power and Energy 221 (2007) 657-668.

[9] M. Heckl, M. Howe, The Rijke Tube: Green's function approach in the time and frequency domain, in: ICSV14, 2007.

[10] S. R. Stow, A. P. Dowling, A Time-Domain Network Model for Nonlinear Thermoacoustic Oscillations, Journal of Engineering for Gas Turbines and Power 131 (2009) 031502.

[11] K. Kashinath, I. C. Waugh, M. P. Juniper, Nonlinear self-excited thermoacoustic oscillations of a ducted premixed flame: bifurcations and routes to chaos, Journal of Fluid Mechanics (761) (2014) $399-430$. 
[12] I. C. Waugh, K. Kashinath, M. P. Juniper, Matrix-free continuation of limit cycles and their bifurcations for a ducted premixed flame, Journal of Fluid Mechanics 759 (2014) 1-27.

[13] H. Mangesius, W. Polifke, A discrete-time, state-space approach for the investigation of non-normal effects in thermoacoustic systems, International Journal of Spray and Combustion Dynamics 3 (4) (2011) 331-350.

[14] A. Orchini, S. J. Illingworth, M. P. Juniper, Frequency domain and time domain analysis of thermoacoustic oscillations with wave-based acoustics, Journal of Fluid Mechanics 775 (2015) 387-414.

[15] A. P. Dowling, A kinematic model of a ducted flame, Journal of Fluid Mechanics 394 (1999) 51-72.

[16] F. Boudy, D. Durox, T. Schuller, G. Jomaas, S. Candel, Describing Function analysis of limit cycles in a multiple flame combustor, Journal of Engineering for Gas Turbines and Power 133 (2011) 061502 .

[17] L. Kabiraj, A. Saurabh, P. Wahi, R. I. Sujith, Route to chaos for combustion instability in ducted laminar premixed flames., Chaos 22 (2012) 023129.

[18] E. Gutmark, K. Wilson, T. Parr, S. K., Feedback control of multi-mode combustion instability, in: 30th AIAA Meeting, 1992.

[19] W. J. Dunstan, R. R. Bitmead, S. M. Savaresi, Fitting nonlinear low-order models for combustion instability control, Control Engineering Practice 9 (2001) 1301-1317.

[20] A. Lamaroui, F. Richecoeur, S. Ducruix, T. Schuller, Experimental analysis of simultaneous nonharmonic related unstable modes in a swirled combustor, in: Proceedings of ASME Turbo Expo 2011, 2011, pp. GT2011-46701.

[21] J. P. Moeck, C. O. Paschereit, Nonlinear interactions of multiple linearly unstable thermoacoustic modes, International Journal of Spray and Combustion Dynamics 4 (1) (2012) 1-28.

[22] M. Cazalens, S. Roux, C. Sensiau, T. Poinsot, Combustion Instability Problems Analysis for HighPressure Jet Engine Cores, Journal of Propulsion and Power 24 (2008) 770-778.

[23] V. V. Anisimov, A. Chiarioni, L. Rofi, C. Ozzano, S. Hermeth, G. Hannebique, G. Staffelbach, T. Poinsot, Bi-stable flame behaviour of heavy duty gas turbine burner. RANS, LES and experiment comparison., in: Proceedings of ASME Turbo Expo, 2015, pp. GT2015-42536.

[24] A. Gelb, W. E. C. Velde, Multiple-input Describing Functions and nonlinear system design, McGraw-Hill, 1968.

[25] F. Selimefendigil, W. Polifke, A nonlinear frequency domain model for limit cycles in thermoacoustic systems with modal coupling, International Journal of Spray and Combustion Dynamics 3 (2011) 303-330.

[26] F. Selimefendigil, S. Foeller, W. Polifke, Nonlinear identification of unsteady heat transfer of a cylinder in pulsating cross flow, Computers and Fluids 53 (2012) 1-14.

[27] A. Orchini, M. P. Juniper, Linear stability and adjoint sensitivity analysis of thermoacoustic networks with premixed flames, Combustion and Flame 165 (2015) 97-108.

[28] L. Kabiraj, R. I. Sujith, Nonlinear self-excited thermoacoustic oscillations: intermittency and flame blowout, Journal of Fluid Mechanics 713 (2012) 376-397.

[29] R. M. Munt, The interaction of sound with a subsonic jet issuing from a semi-infinite cylindrical pipe, Journal of Fluid Mechanics 83 (4) (1977) 609-640.

[30] A. M. Cargill, Low frequency acoustic radiation from a jet pipe - a second order theory, Journal of Sound and Vibration 83 (1982) 339-354.

[31] M. C. A. Peters, A. Hirschberg, A. J. Reijnen, A. P. J. Wijnands, Damping and reflection coefficient measurements for an open pipe at low Mach and low Helmholtz numbers, Journal of Fluid Mechanics 256 (1993) 499-534.

[32] A. P. Dowling, S. R. Stow, Acoustic analysis of gas turbine combustors introduction, Journal of Propulsion and Power 19 (5) (2003) 751-764.

[33] G. H. Markstein, Non-steady flame propagation, Pergamon Press, 1964.

[34] S. Hemchandra, Preetham, T. Lieuwen, Response of turbulent premixed flames to harmonic acoustic forcing, Proceedings of the Combustion Institute 31 (2007) 1427-1434.

[35] K. Kashinath, S. Hemchandra, M. P. Juniper, Nonlinear phenomena in thermoacoustic systems with premixed flames, Journal of Engineering for Gas Turbines and Power 135 (6) (2013) 061502.

[36] A. L. Birbaud, D. Durox, S. Candel, Upstream flow dynamics of a laminar premixed conical flame submitted to acoustic modulations, Combustion and Flame 146 (2006) 541-552.

[37] K. Kashinath, S. Hemchandra, M. P. Juniper, Nonlinear thermoacoustics of ducted premixed flames: The influence of perturbation convection speed, Combustion and Flame 160 (2013) 2856-2865.

[38] J. A. Sethian, Level Set methods and Fast Marching methods, Cambridge University Press, 1999.

[39] D. Hartmann, M. Meinke, W. Schröder, A level-set based adaptive-grid method for premixed combustion, Combustion and Flame 158 (2011) 1318-1339. 
[40] F. Baillot, D. Durox, R. Prud'Homme, Experimental and theoretical study of a premixed vibrating flame, Combustion and Flame 88 (1992) 149-168.

[41] Preetham, T. Lieuwen, Dynamics of laminar premixed flames forced by harmonic velocity disturbances, Journal of Propulsion and Power 24 (6) (2008) 1390-1402.

[42] D. Durox, T. Schuller, N. Noiray, S. Candel, Experimental analysis of nonlinear Flame Transfer Functions for different flame geometries, Proceedings of the Combustion Institute 32 (2009) 13911398.

[43] T. Schuller, S. Ducruix, D. Durox, S. Candel, Modeling tools for the prediction of premixed Flame Transfer Functions, Proceedings of the Combustion Institute 29 (2002) 107-113.

[44] Shreekrishna, S. Hemchandra, T. Lieuwen, Laminar premixed flame response to equivalence ratio oscillations, Combustion Theory and Modelling 14 (5) (2010) 681-714.

[45] A. Cuquel, D. Durox, T. Schuller, Theoretical and experimental determination of the Flame Transfer Function of confined premixed conical flames, in: 7th Mediterranean Combustion Symposium, 2011.

[46] S. Hemchandra, Premixed flame response to equivalence ratio fluctuations: comparison between reduced order modeling and detailed computations, Combustion and Flame 159 (2012) 3530-3543.

[47] S. Ducruix, D. Durox, S. Candel, Theoretical and experimental determinations of the Transfer Function of a laminar premixed flame, Proceedings of the Combustion Institute 28 (1) (2000) 765773.

[48] N. Karimi, M. J. Brear, S. Jin, J. P. Monty, Linear and non-linear forced response of a conical, ducted, laminar premixed flame, Combustion and Flame 156 (11) (2009) 2201-2212.

[49] M. Juniper, L. Li, J. Nichols, Forcing of self-excited round jet diffusion flames, Proceedings of the Combustion Institute 32 (2008) 1191-1198.

[50] L. Tay-Wo-Chong, S. Bomberg, A. Ulhaq, T. Komarek, W. Polifke, Comparative Validation Study on Identification of Premixed Flame Transfer Function, Journal of Engineering for Gas Turbines and Power 134 (021502).

[51] M. Hoeijmakers, V. Kornilov, I. L. Arteaga, P. de Goey, H. Nijmeijer, Intrinsic instability of flameacoustic coupling, Combustion and Flame 161 (2014) 2860-2867.

[52] T. Emmert, S. Bomberg, W. Polifke, Intrinsic thermoacoustic instability of premixed flames, Combustion and Flame (162) (2014) 75-85.

[53] M. Hoeijmakers, V. Kornilov, I. L. Arteaga, P. de Goey, H. Nijmeijer, Flames in context of thermoacoustic stability bounds, Proceedings of the Combustion Institute 35 (1) (2014) 1073-1078.

[54] E. Courtine, L. Selle, T. Poinsot, DNS of intrinsic thermoacoustic modes in laminar premixed flames, Combustion and Flame 162 (2015) 4331-4341.

[55] T. Schuller, D. Durox, S. Candel, A unified model for the prediction of laminar Flame Transfer Functions: comparisons between conical and V-flames dynamics, Combustion and Flame 134 (1-2) (2003) 21-34.

[56] M. Schmid, R. S. Blumenthal, M. Schulze, W. Polifke, T. Sattelmayer, Quantitative S-tability Analysis Using Real-Valued Frequency Response Data, Journal of Engineering for Gas Turbines and Power 135 (2013) 121601.

[57] X. Han, J. Li, A. S. Morgans, Prediction of combustion instability limit cycle oscillations by combining flame describing function simulations with a thermoacoustic network model, Combustion and Flame 162 (2015) 3632-3647.

[58] J. Guckenheimer, P. Holmes, Nonlinear oscillations, dynamical systems and bifurcations of vector fields, Springer, 1983. 